\title{
Air cushioning with a lubrication/inviscid balance
}

\author{
By F. T. SMITH ${ }^{1}$, L. LI ${ }^{1}$ AND G. X. W U ${ }^{2}$ \\ ${ }^{1}$ Department of Mathematics, University College London, Gower Street, London WC1E 6BT, UK \\ ${ }^{2}$ Department of Mechanical Engineering, University College London, Gower Street, \\ London WC1E 6BT, UK
}

(Received 24 September 2001 and in revised form 19 December 2002)

The air cushioning effect in the gap between an almost inviscid body of water and a nearby solid wall (or another body of water) is studied theoretically and is found to depend on predominantly lubricating forces in the air, in certain applications. The situation in which the density and viscosity in air are taken as small compared with those in water is investigated. In this situation potential-flow dynamics in the water couples with lubrication behaviour in the air, leading to a nonlinear integro-differential system for the evolution of the interface. The numerical values of the main parameters are investigated and indicate a wide range of practical applications. Specifically, the lubrication/inviscid balance holds for typical global Reynolds numbers below the order of the viscosity ratio divided by the cube of the density ratio, i.e. below about $10^{7}$ in the case of air and water; for Reynolds numbers of that order the lubrication behaviour is replaced by an unsteady boundary-layer response, whereas above that order formally the response is totally inviscid. A variety of spatio-temporal flow solutions are presented for the lubrication/inviscid system and these all indicate a relatively rapid closure of the gap, in a common form which is analysed.

\section{Introduction}

The present work considers an almost inviscid body of fluid (water) which is bounded by a free surface, is approaching impact with a solid wall or another such body of fluid and is gradually affected by the 'cushioning' fluid (air) in-between. Most studies on this pre-impact stage and the subsequent post-impact stage examine totally inviscid or solely viscous slow-flow effects locally, whether the cushioning fluid is included or not. These studies include very interesting groupings of papers in the totally inviscid case by Korobkin \& Peregrine (2000), Walkden et al. (2000), Wood \& Peregrine (2000), Wood, Peregrine \& Bruce (2000); by Howison, Ockendon \& Wilson (1991), Wilson (1991), Howison, Ockendon \& Oliver (2002); by Lesser \& Field (1983), Korobkin (1997, 1999); and by Zhao \& Faltinsen (1993), Cooker (1997), Cooker, Weidman \& Bale (1997). Here, Wood et al. and others address the approximations of pressure-impulse theory for water alone in an impact, while of special relevance to the current investigation are the theoretical works by Howison et al. and Wilson on thin-layer dynamics of cushioning and Korobkin on compressibility effects, the latter effects having been reviewed earlier by Lesser \& Field (1983). Typically, potential flow properties in the water are coupled with inviscid thin-layer responses in the air. See also the thin-layer analyses in Joseph \& Preziosi (1987), Kelmanson (1995), Oron, Davis \& Bankoff (1997), Weidner, Schwartz \& Eres (1997), Duffy \& Wilson (1999), 
Davis (2000) concerning the mainly viscous case. Other studies addressing surface tension effects are by Kriegsmann, Miksis \& Vanden-Broeck (1998) and McKinley \& Wilson (2001) using lubrication theory, Miksis \& Vanden-Broeck (1999) on slow flow and Kang \& Vanden-Broeck (2000) on inviscid flow theory, and helpful reviews on different aspects are included in Yarin \& Weiss (1995), Quéré (1999), Weiss \& Yarin (1999), Zhu, Oguz \& Prosperetti (2000), Barannyk \& Papageorgiou (2002), with Rein (1993) concentrating in particular on post-impact phenomena.

There is a strong possibility, however, that the cushioning may mainly involve a combined viscous-inviscid effect such that a balance between lubrication forces in the air and inviscid pressure forces in the water is produced. This is partly because the air density $\rho_{2}$ and viscosity $\mu_{2}$, which play important roles in determining the effective pressure and shear stress, are quite small compared with the corresponding water quantities $\rho_{1}$ and $\mu_{1}$. Representative values of the ratios $\rho_{2} / \rho_{1}$ and $\mu_{2} / \mu_{1}$ are $1 / 828$ and $1 / 55$, respectively, implying that the ratio of the kinematic viscosities $\nu_{2} / \nu_{1}$ is about 15. [At $20^{\circ} \mathrm{C}$, dry air at one-atmosphere pressure has $\rho_{2}, \mu_{2}, v_{2}$ values of $1.205 \times$ $10^{-3}, 1.81 \times 10^{-4}, 0.150$, respectively, whereas pure water has $\rho_{1}, \mu_{1}, v_{1}$ equal to 0.9982 , $1.002 \times 10^{-2}, 1.004 \times 10^{-2}$ in turn, in c.g.s. units.] Thus the density and viscosity ratios can within reason be considered small, with the kinematic viscosity ratio being either moderately large or of order unity, when comparisons between inertial and viscous forces (involving local Reynolds numbers for instance) are being made in either fluid. The discussion herein is mostly with the water and air combination in view, but it applies more generally to two fluids with small ratios of density and viscosity and a moderately large or $O(1)$ ratio of kinematic viscosity. We note also that the work here stemmed from a particular configuration with a nearly vertical air gap which is thin initially, as studied by Li et al. $(2001,2002)$, e.g. on the side of a floating body, but the resulting argument turns out to be generic, holding in principle for air gaps which need not be initially thin or vertical. A similar lubrication/inviscid balance is investigated by King \& Tuck (1993), King, Tuck \& Vanden-Broeck (1993), Vanden-Broeck \& Miloh (1996), Vanden-Broeck (2001) and others, in various settings, for steady or travelling waves rather than the spatio-temporal evolution of present concern. The relevant contexts may include applications to the air pockets trapped under the keel of a ship as in the experiments of Nethercote, Mackay \& Menon (1986) (described by Howison et al. 1991, Wilson 1991, see also Driscoll \& Lloyd 1982 ) or as in the experiments in Lesser \& Field (1983), the impact experiments described by Wood et al. (2000) and references therein, and the high pressures induced when island landslides enter the sea (e.g. see Ward 2001; Ward \& Day 2001), among other splashing applications. In any case the numerical values are found, later in the paper, to be such that the cushioning by a thin air gap can fairly readily involve viscous or inviscid forces, or indeed both, depending on the precise local setting.

The setting is taken to be two-dimensional and can be described through the Cartesian coordinates $x^{*}, y^{*}$, where the superscript * signifies a dimensional quantity. The velocity components in the $x^{*}$-, $y^{*}$-directions are written $u^{*}, v^{*}$, respectively. The full conditions at the unknown interface between the two fluids then require the normal and tangential components of stress to be continuous, the tangential velocity component to be continuous and the kinematic constraint on normal velocity to be satisfied in each fluid. In the cases of present concern the configurations locally are slender. The shear stress and the tangential velocity on the interface can be approximated by $\mu \partial u^{*} / \partial y^{*}$ and $u^{*}$ in turn. They are required to be continuous together with the pressure $p^{*}$. The kinematic condition is applied to the normal 
velocity which is approximately $v^{*}$. Here $\mu=\mu_{1}, \mu_{2}$ and $y^{*}$ is essentially the normal coordinate. An example is the floating-body case of Li et al. mentioned earlier whose vertical air gap starts thin, of a shape given by the side free surface which is controlled by the overall effective body contour and displacement, whereas the upper free surface remains almost horizontal. The thinness of the air gap is responsible, when combined with the density and viscosity ratios, for the subsequent viscous rather than inviscid thin-layer effect as impact is approached, whether with a solid surface or another body of fluid.

The physical argument is presented first in $\S 2$ for a variety of local configurations involving relatively thin air gaps (see figure $1 a$ ). The air velocities greatly exceed those of the water in general, but the mass flux in the air gap is relatively small and in addition a passive shear layer is generated close to the interface. A detailed examination of the numerical values of the parameters involved is given in $\S 3$, which indicates a wide range of potential practical applications. The argument is applied in $\S 4$ to the specific case of a floating body plunging downwards, where typically no slip holds on a downward moving solid surface on one side of the air gap as in figure $1(b)$, in contrast with the interface on the other side. We observe that in effect the coordinate $x^{*}$ is horizontal in figure $1(a)$ but vertical in figure $1(b)$. The local reasoning presented in $\S \S 2$ and 4 leads to the main controlling equations. These couple in essence the potential-flow (water) and lubrication (air) relations between the unknown evolving interfacial pressure and shape. Spatio-temporal flow solutions obtained computationally under various conditions are described in $\S 5$, where it is found that an approaching touchdown phenomenon (in which the scaled air-gap thickness tends to zero) is characteristic of the solutions, and this phenomenon is analysed. Further comments and conclusions are provided in $\S 6$, including an enhanced system (namely the unsteady boundary-layer equations), its applicability in air cushioning and its break-up, along with discussion of compressibility effects and experiments.

\section{The general local setting, and the main controlling equations}

Suppose initially that a body of water is in motion relatively far from a planar wall, with air in between. If the water flow has a typical Reynolds number of order unity, the smallness of the viscosity and density ratios $\mu_{2} / \mu_{1}, \rho_{2} / \rho_{1}$ implies that the air is dynamically negligible as a valid first approximation. So the Navier-Stokes equations can be solved in the water subject to classical free-surface conditions of constant pressure and zero tangential stress at the water-air interface. This, together with other relevant boundary conditions elsewhere in the flow, determines the evolution of the free surface and the tangential velocity of the water at that surface. The air motion can then be determined to leading order by solving subject to prescribed velocity conditions at the prescribed interface shape, given by the water solution above. The tangential stress and normal stress, in particular the air pressure, generated in the air motion at the interface then serve as a leading-order correction for the water-flow calculation, and so on. (Strictly of course this is for two model fluids with ratios $\mu_{2} / \mu_{1}, \rho_{2} / \rho_{1}$ tending to zero). If the Reynolds number is increased sufficiently, the water motion above becomes virtually inviscid except for a thin interfacial layer generated by tangential stresses; we ignore here any contact with a solid surface elsewhere in the flow which could cause separation. Although the air motion likewise may become inviscid there is no significant effect yet from the air motion on the water flow or on the interfacial shape. 
(a)
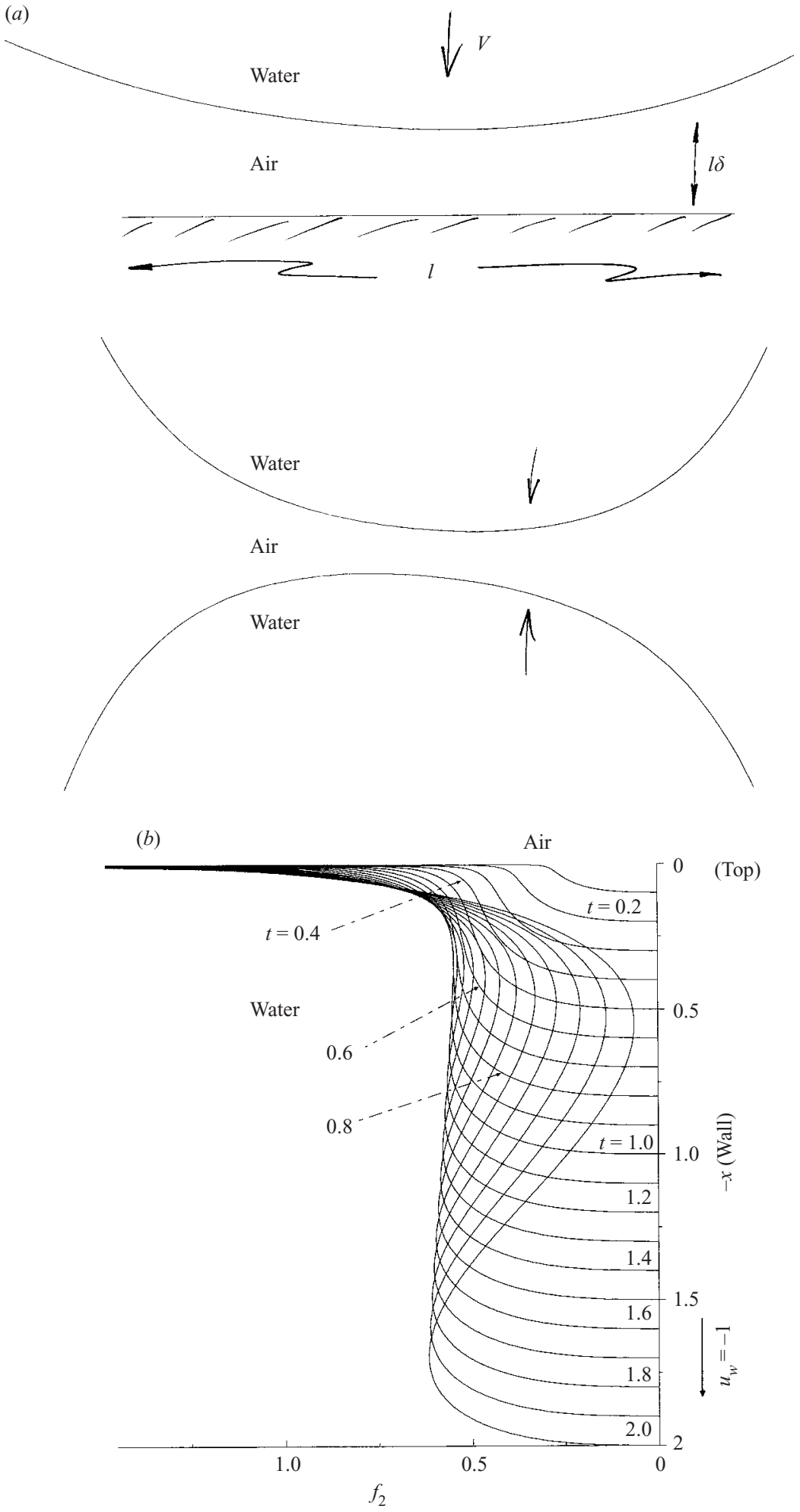

FIGURE 1. For caption see facing page. 
Suppose now that the body of water approaches the wall. The characteristic shape of the interface just prior to any first contact with the wall is a parabola, but in any case a thin gap containing air is created. The response of the air motion in that gap can be of predominantly inviscid thin-layer form or viscous-inviscid (boundary layer) form or viscous (lubrication) form, depending on the viscosity and density ratios and their interplay with the local Reynolds number of the air flow, and is expected to provoke enhanced air pressures and maximum velocities through the gap. This response is akin to the so-called ground effect studied theoretically by Tuck \& Bentwich (1983), Wilson \& Duffy (1998), Jones (2000), Purvis (2002) and Jones \& Smith (2003) among others, as well as to the thin-layer responses mentioned in $\S 1$. The enhanced pressures here produce air cushioning in the sense that when they grow to become comparable with the typical water pressures locally then the water motion and specifically the interface shape is altered substantially in the vicinity of the air gap. There is little question that the governing equations in the air motion at that cushioning stage should be expected to be those of a lubricating layer, boundary layer or inviscid boundary layer, and our interest below will be mostly in the lubricating case. The more significant question is when and how the cushioning takes place, and for that the detailed scales, matching and boundary conditions are to be investigated. For instance, the air velocities are found to exceed those in the water by an order of magnitude, when cushioning occurs, indicating that the scaled air velocity must tend to zero at the unknown interface, for matching purposes. The physical setting is similar to that above if two bodies of water approach each other.

The local argument near an impact as presented below is relatively simple because only two main flow regions are involved; there is also an in-between shear layer at the water-air interface, but it is negligible, as a specific case later shows. The central reasoning and resultant equations are given in $\$ 2.1$, based on the Navier-Stokes equations in dimensional form

$$
\rho_{n}\left(\frac{\partial}{\partial t^{*}}+\boldsymbol{u}_{\mathbf{n}}^{*} \cdot \nabla\right) \boldsymbol{u}_{\boldsymbol{n}}^{*}=-\nabla p_{n}^{*}-\rho_{n}(0, g)+\mu_{n} \nabla^{2} \boldsymbol{u}_{\mathbf{n}}^{*},
$$

for $n=1,2$; the subscripts 1 and 2 where used refer to quantities in water and air, respectively. The Cartesian coordinate $x^{*}$ is horizontal here, as in figure 1( $\left.a\right), g$ denotes the acceleration due to gravity, $t^{*}$ is time, $\boldsymbol{u}^{*}$ is $\left(u^{*}, v^{*}\right)$ and $\nabla$ is the operator $\left(\partial / \partial x^{*}, \partial / \partial y^{*}\right)$. The continuity equation requires $\nabla \cdot \boldsymbol{u}^{*}=0$. Further application is discussed in $\S 2.2$. Figure $1(a)$ is relevant to this section, whereas figure $1(b)$ is relevant to $\S 4$.

\subsection{The local argument and governing equations}

As impact is approached, suppose the characteristic local length scale of the water is $\ell$ in the direction parallel to the solid wall and the air gap-width scale is $\ell \delta$, with the fraction $\delta$ taken to be small, while the typical approach speed of the water toward the wall is $V$. Then the major part of the water flow is expected to have $x^{*} \sim y^{*} \sim \ell$, a short time scale $t^{*} \sim \gamma_{1} \ell / V$ where the small fraction $\gamma_{1}$ is to be found, velocities $u_{1}^{*} \sim v_{1}^{*} \sim V$ and pressure $p_{1}^{*}$ of size $\rho_{1} V^{2} / \gamma_{1}$ to balance the flow

FiguRE 1. The flow configurations: $(a)$ the general local setting examined in $\S \S 2$ and 3 and shown here in dimensional terms with air cushioning between water and solid or between two bodies of water; $(b)$ with a free surface close to a downward plunging solid surface (wall), as in $\S 4$, in non-dimensional form. 
acceleration. The relatively high pressure gradients generated in the water motion and hence in the air are consistent with high acceleration/deceleration in the water as the typical velocities $u_{1}^{*}, v_{1}^{*}$ change from the given non-zero value $V$ towards zero (say) over the short time scale involved. The coordinates $x^{*}$ along the plate and $y^{*}$ normal have their origin in the impact zone. From (2.1), the governing equations are then inviscid and gravity-free,

$$
\frac{\partial u_{1}^{*}}{\partial x^{*}}+\frac{\partial v_{1}^{*}}{\partial y^{*}}=0, \quad \rho_{1} \frac{\partial u_{1}^{*}}{\partial t^{*}}=-\frac{\partial p_{1}^{*}}{\partial x^{*}}, \quad \rho_{1} \frac{\partial v_{1}^{*}}{\partial t^{*}}=-\frac{\partial p_{1}^{*}}{\partial y^{*}},
$$

provided that the Froude number $\mathscr{G}\left(\equiv V^{2} / g \ell\right)$ and Reynolds number $r e_{1}\left(\equiv V \ell / v_{1}\right)$ based on local quantities are both much greater than $\gamma_{1}$. These and other assumptions are checked later in this section. Here we use $r e_{1}, r e_{2}$ to denote local Reynolds numbers in the water and air, in turn, and reserve $R e_{1}, R e_{2}$ for the corresponding global quantities. The smallness of $\gamma_{1}$, i.e. the assumption that the time scale is relatively short, rules out nonlinear inertial effects compared with the relatively high acceleration/deceleration effects here. The kinematic and dynamic conditions on $v^{*}=v_{1}^{*}, p^{*}=p_{1}^{*}$ at the interface become

$$
v^{*} / V \rightarrow F_{T}, \quad \gamma_{1} p^{*} /\left(\rho_{1} V^{2}\right) \rightarrow P,
$$

as $y^{*} \rightarrow 0+$, where the shape of the interface $y^{*} / \ell=\gamma_{1} F(X, T)$, with $X \equiv x^{*} / \ell$ and $T \equiv V t^{*} /\left(\gamma_{1} \ell\right)$, is to be found. The scaled pressure $P\left[\equiv \gamma_{1} p^{*} /\left(\rho_{1} V^{2}\right)\right.$ evaluated on the interface] is also an unknown function of the scaled coordinates $X, T$. Since $\nabla^{2} p^{*}=0$, the solution of $(2.2 a-d)$ subject to far-field boundedness can be obtained through an analytical complex function $p^{*}+\mathrm{i} q^{*}$ in the complex plane $z^{*}=x^{*}+\mathrm{i} y^{*}$. Cauchy's integral theorem gives

$$
\frac{\mathrm{d}}{\mathrm{d} z^{*}}\left(p^{*}+\mathrm{i} q^{*}\right)=\frac{1}{2 \pi \mathrm{i}} \int_{-\infty}^{\infty} \frac{\left[p_{\xi}^{*}\left(\xi, 0, t^{*}\right)+\mathrm{i} q_{\xi}^{*}\left(\xi, 0, t^{*}\right)\right] \mathrm{d} \xi}{\left(\xi-z^{*}\right)}
$$

and so, from the imaginary parts evaluated at $y^{*}=0$,

$$
\frac{\partial p^{*}}{\partial y^{*}}\left(x^{*}, 0, t^{*}\right)=\frac{1}{\pi} f_{-\infty}^{\infty} \frac{p_{\xi}^{*} \mathrm{~d} \xi}{\left(\xi-x^{*}\right)} .
$$

Using $(2.2 a-d)$ in the scaled coordinates $X$ and $T$ we thus have the relation

$$
F_{T T}(X, T)=\frac{1}{\pi} f_{-\infty}^{\infty} \frac{P_{Q}(Q, T) \mathrm{d} Q}{X-Q}
$$

between the unknown scaled interface shape and pressure.

In the air gap, $y^{*} \sim \ell \delta$ is generally smaller than in the water, whereas $x^{*} \sim \ell$ $(X \sim 1)$ still, and $v_{2}^{*} \sim V$ from the interface condition. So mass conservation suggests $u_{2}^{*} \sim V / \delta$ must be relatively large, while the matching of the pressure requires the order of magnitude of $p^{*}=p_{2}^{*}$ here to be identical to that $\left(p_{1}^{*}\right)$ of the water motion. (The scale $V / \delta$ of the air velocities $u_{2}^{*}$ induced in the gap is assumed to be not so large as to introduce significant compressibility effects within the present stage, see $\S 6$.) The governing equations in the air are

$$
\frac{\partial u_{2}^{*}}{\partial x^{*}}+\frac{\partial v_{2}^{*}}{\partial y^{*}}=0, \quad 0=-\frac{\partial p_{2}^{*}}{\partial x^{*}}+\mu_{2} \frac{\partial^{2} u_{2}^{*}}{\partial y^{* 2}}, \quad 0=-\frac{\partial p_{2}^{*}}{\partial y^{*}}
$$

from (2.1), i.e. those of classical incompressible lubrication theory, provided that acceleration, inertial and gravity forces are negligible compared with the pressure 
gradient force in $(2.4 b)$. The latter three requirements are that $\rho_{2} V^{2} /\left(\delta \gamma_{1} \ell\right), \rho_{2} V^{2} /\left(\delta^{2} \ell\right)$, $\rho_{2} g$ are all much less than $\rho_{1} V^{2} /\left(\gamma_{1} \ell\right)$, while the pressure-gradient/viscous balance holds for $\rho_{1} V^{2} /\left(\gamma_{1} \ell\right) \sim \mu_{2} V /\left(\ell^{2} \delta^{3}\right)$. The lateral pressure gradient in $(2.4 c)$ must be zero if $\rho_{1} V^{2} /\left(\gamma_{1} \delta \ell\right)$ is much larger than $\rho_{2} V^{2} /\left(\gamma_{1} \ell\right), \rho_{2} V^{2} /(\delta \ell), \mu_{2} V /\left(\delta^{2} \ell^{2}\right)$; gravity effects are reconsidered later. The condition on $\hat{y}=F$ for the air is obtained by taking into account a thin shear layer in the water at the interface. This thin layer has little effect on $(2.2 a-c)$ but it plays a not insignificant role in the air-water interaction by smoothing the velocity and stress responses locally. Since $y^{*}=\ell \gamma_{1} F$ is relatively small, the conditions on the interface based on the continuity of velocity and shear force can be written as

$$
u_{1}^{*}=u_{2}^{*}, \quad \mu_{1} \frac{\partial u_{1}^{*}}{\partial y^{*}}=\mu_{2} \frac{\partial u_{2}^{*}}{\partial y^{*}}, \quad v_{1}^{*}=v_{2}^{*} .
$$

In the scaled system defined by

$$
u_{2}^{*}=\frac{V}{\delta} \hat{u}, \quad v_{2}^{*}=V \hat{v}, \quad p_{2}^{*}=\frac{\rho_{1} V^{2} P}{\delta}, \quad y^{*}=\ell \delta \hat{y},
$$

these conditions can be approximated as

$$
\hat{u}=0, \quad \hat{v}=\partial F / \partial T \quad \text { at } \quad \hat{y}=F,
$$

while the no-slip condition on the wall requires

$$
\hat{u}=\hat{v}=0 \quad \text { at } \quad \hat{y}=0 .
$$

All of these are the same as in $(4.5 a-d)$ below in normalized form with $\gamma_{1}$ now identified with $\delta$ for matching. The solution for the air flow can be found by writing $u_{2}^{*}$ as a parabolic function of $y^{*}$ because of $(2.4 b, c)$, with coefficients determined by the conditions on $u_{2}^{*}$ at $y^{*}=0$ and $y^{*}=\ell \gamma_{1} F$. Substituting $u_{2}^{*}$ into $(2.4 a)$, integrating the result with respect to $y^{*}$ from 0 to $\ell \gamma_{1} F$ and using the conditions on $v_{2}^{*}$ at $y^{*}=0$ and $y^{*}=\ell \gamma_{1} F$, we obtain the Reynolds lubrication equation in the scaled system,

$$
\left(F^{3} P_{X}\right)_{X}=12 \Gamma F_{T}
$$

where $\Gamma$ denotes $\mu_{2} /\left(\delta^{2} \rho_{1} V \ell\right)$. Thus a coupled problem $(2.3),(2.5)$ for $F, P$ is obtained. The fact that the water is initially moving towards the wall imposes a condition of the form $F \sim \hat{a}_{2} X^{2}-T$ (in keeping with $v^{*} \rightarrow-V, \hat{v} \rightarrow-1$ as well as with (2.3), (2.5)) for large negative scaled times $T$. This is due to the incident parabolic shape of the interface. The constant $\hat{a}_{2}$ is positive. Further, the requirement that the parameter $\Gamma$ can be regarded as $O(1)$ is equivalent to the local water Reynolds number $r e_{1}$ being of order $\mu_{2} /\left(\mu_{1} \delta^{2}\right)$ since $r e_{1}$ is $V \ell \rho_{1} / \mu_{1}$; here we will take the ratio $\nu_{2} / \nu_{1}$ to be $O(1)$ or just numerically large, whereas $\rho_{2} / \rho_{1}(\approx 1 / 828), \mu_{2} / \mu_{1}(\approx 1 / 55)$ are both considered small parameters.

Integro-differential equations similar to but different from (2.3), (2.5) are derived in Fitt \& Pope (2001) and related papers in the context of the unsteady movement of sails and flags and with less emphasis on spatio-temporal evolution than in the present work. Again see the King \& Tuck (1993), King et al. (1993), Vanden-Broeck $\&$ Miloh (1996) papers described in $\S 1$ for other lubrication/inviscid settings.

We consider now the assumptions behind (2.2)-(2.4) in non-dimensional form. The orders of magnitude give

$$
\gamma_{1} / \delta \sim 1, \quad r e_{1}=V \ell / \nu_{1} \sim \mu_{2} /\left(\mu_{1} \delta^{2}\right)
$$


These determine all the scales above. The inequalities used require $\delta, \mathscr{G}$ to satisfy

$$
\begin{gathered}
\frac{\rho_{2}}{\rho_{1}} \ll \delta \ll \frac{\rho_{1}}{\rho_{2}}, \quad \delta \ll\left(\frac{\mu_{2}}{\mu_{1}}\right)^{1 / 3}, \\
\mathscr{G} \gg \delta, \quad \mathscr{G} \gg\left(\frac{\rho_{2}}{\rho_{1}}\right) \delta .
\end{gathered}
$$

These assumptions are satisfied fairly readily for air and water (see the ratio values in $\S 1$ ), with a small $\delta$, a Froude number which needs only to be large relative to $\delta$, and local water and air Reynolds numbers $r e_{1}, r e_{2}\left(=V \ell / v_{1}, V \ell / v_{2}\right)$ which are comparable with $\mu_{2} /\left(\mu_{1} \delta^{2}\right), \rho_{2} /\left(\rho_{1} \delta^{2}\right)$ or $1 /\left(55 \delta^{2}\right), 1 /\left(828 \delta^{2}\right)$ respectively, from (2.6), and which need not be excessively large or small.

The asymptotic expansions corresponding to the above reasoning are given in the Appendix. One property which we will use later concerns the characteristic global Reynolds number $R e_{1}$ (in the water) which must be distinguished clearly from the local Reynolds number. Since the interface curvature $\mathrm{d}^{2} y^{*} / \mathrm{d} x^{* 2}$ is of the order $\delta / \ell$, the typical radius of curvature $r$ of the water body is $\ell / \delta$. Hence $R e_{1}$, if based on $V$ and $r$, is approximately the local Reynolds number $r e_{1}$ multiplied by $1 / \delta$ (likewise for the air motion, $R e_{2}$ is approximately $r e_{2} / \delta$ ). Moreover this multiplicative factor is enhanced if the main velocity component of the water body is parallel to the solid surface as in $\S 4$ below and in figure $1(b)$.

\subsection{Enlarging the range of applications}

In this subsection we consider a number of extensions to the theory.

If, instead of a solid wall, the impending collision is between two equal but opposing bodies of water, then the no-slip condition just after $(2.4 c)$ is replaced by the symmetry condition $\partial \hat{u} / \partial \hat{y}=\hat{v}=0$ at $\hat{y}=0$, in effect. The latter leads to the factor $12 \Gamma$ being replaced by $3 \Gamma$ in (2.5) and hence to the same normalized problem.

If the body of water approaching a solid wall is of more general shape, the boundary conditions on $F, P$ have the form

$$
F \sim A(X) T+B(X), \quad P \rightarrow 0 \quad \text { as } \quad T \rightarrow-\infty,
$$

which satisfies (2.3) and (2.5). There are certain restrictions on the shape functions $A, B$. In the particular case of an approaching parabola in $\S 4 A, B$ are $-b_{2}$ and $a_{2} X^{2}$, respectively.

If the collision is between two general shapes, then let their scaled interfaces be at $y^{*} /(\ell \delta)=F_{1}$ and $-F_{2}$ say, measured from the Cartesian $x^{*}$ axis. The gap width $F_{1}+F_{2}$ still satisfies $(2.5)$, from solving $(2.4 a-c)$ with the appropriate pair of conditions at each interface $\hat{y}=F_{1}, \hat{y}=-F_{2}$, but on the other hand each of $\partial^{2} F_{1} / \partial T^{2}, \partial^{2} F_{2} / \partial T^{2}$ is given by a pressure integral as in (2.3), from the separate bodies of water. Since the pressures must be equal (due to $(2.4 c)$ ) so must the two double derivatives in $T$. Hence $F_{1}+F_{2}$ also satisfies (2.3) apart from a factor 2 and the pair $\left(F_{1}+F_{2}, P\right)$ satisfies the same system as the original $(F, P)$. The difference $F_{1}-F_{2}$, which has to have the form $C(X) T+D(X)$ for all $T$ as its double derivative with respect to $T$ remains equal to zero throughout, with functions $C, D$ fixed by the initial conditions, determines the movement of the whole water-air-water combination.

If the gravity force is stronger than supposed in $\S 2.1$ (and similarly in $\S 4$ later), or if there is substantial pre-existing flow acceleration, or both, then each of (2.3), (2.5) is altered. Simultaneously, an inclination to the vertical can be accounted for. The gravity force when the wall is inclined to the vertical can be represented by the 
effective Froude numbers $\mathscr{F}, \mathscr{G}$ in the $x^{*}-, y^{*}$-directions respectively. For these to have significant effect on the inviscid/lubrication balance described so far, $\mathscr{F}, \mathscr{G}$ must be sufficiently small, e.g. from increased gravity, that the inviscid equations of the water region are modified, and likewise for the strong lubrication forces in the air motion. In fact the orders of magnitude of the forces in the water flow suggest at first that $\mathscr{F}$, $\mathscr{G}$ values of order $\delta^{2}$ are required, while relatively large incident accelerations $\partial u_{1}^{*} / \partial t^{*}$ of order $V^{2} /(\ell \mathscr{F})$ can be accounted for. The large inverse Froude number $\mathscr{F}^{-1}$ is expanded in the form $\delta^{-2} \tilde{\mathscr{F}}^{-1}+\delta^{-1} \hat{\mathscr{F}}^{-1}$ say (so the Froude number is small enough to be comparable at leading order with the square of the small interfacial slope locally). This decomposition effect is taken to contribute both an extra term $\kappa_{2} X \hat{\mathscr{F}}-1$ to be scaled pressure and an extra term $-(T-\tilde{c}) \delta^{-1} \tilde{\mathscr{F}}^{-1}-\kappa_{1}(T-\tilde{c}) \hat{\mathscr{F}}^{-1}$ to the scaled $u^{*}$ representing an underlying $x^{*}$-motion, because all of the constant gravity force $\mathscr{F}^{-1}$ can be balanced by constant pressure gradient $\partial p^{*} / \partial x^{*}$ or constant pre-existing acceleration $\partial u_{1}^{*} / \partial t^{*}$ acting in the incident water flow; see $(2.2 a-c)$. Here $\tilde{c}, \kappa_{1}, \kappa_{2}$ are constants and $\kappa_{1}=\kappa_{2}+1$. Because of the inclination to the vertical, the effective inverse Froude number $\mathscr{G}^{-1}$ in the $y^{*}$-direction is decomposed as $\delta^{-2} \tilde{\mathscr{G}}^{-1}+\delta^{-1} \hat{\mathscr{G}}^{-1}$, similarly contributing $\sigma_{2} y^{*} \ell^{-1} \hat{\mathscr{G}}^{-1}$ to pressure and $-(T-\tilde{c}) \delta^{-1} \tilde{\mathscr{G}}^{-1}-\sigma_{1}(T-\tilde{c}) \hat{\mathscr{G}}^{-1}$ to $v_{1}^{*}$, with constants $\sigma_{1}=\sigma_{2}+1$; however $\tilde{\mathscr{G}}^{-1}$ must in fact be zero for consistency in the present balance within the kinematic condition. Thus the incident water-flow solution prior to any significant air-cushioning taking effect has the form

$$
\begin{gathered}
u_{1}^{*} / V=-\left(T-\tilde{c}_{1}\right) /(\delta \tilde{\mathscr{F}})-\kappa_{1}\left(T-\tilde{c}_{2}\right) / \hat{\mathscr{F}}+u_{E}, \\
v_{1}^{*} / V=-\sigma_{1}\left(T-\tilde{c}_{3}\right) / \hat{\mathscr{G}}+v_{E}, \\
p_{1}^{*} \delta /\left(\rho_{1} V^{2}\right)=\kappa_{2} X / \hat{\mathscr{F}}+\sigma_{2} y^{*} /(\ell \hat{\mathscr{G}})+p_{E},
\end{gathered}
$$

showing the acceleration or deceleration of the body of water and the pressure gradients, with $u_{E}, v_{E}, p_{E}$ being zero. Here $\tilde{c}_{1}, \tilde{c}_{2}, \tilde{c}_{3}$ are arbitrary constants and we take the special case $\tilde{c}_{1}=\tilde{c}_{2}=\tilde{c}_{3}(=\tilde{c})$ for convenience. The subsequent air cushioning interaction, however, then makes $u_{E}, v_{E}, p_{E}$ become non-zero and of order unity locally, in the water motion, giving a nonlinear perturbation to the above incident form rather than the case of zero $\tilde{\mathscr{F}}^{-1}, \hat{\mathscr{F}}^{-1}, \hat{\mathscr{G}}^{-1}$ considered in the previous subsection but with all scalings and assumptions otherwise being as described there. This introduces into the momentum equations additional inertial effects comparable with the unsteady responses in $(2.2 b, c)$ during cushioning, since now $\partial / \partial t^{*} \sim u_{1}^{*} \partial / \partial x^{*}$ at leading order. Similarly, the right-hand sides in the kinematic and normal-stress conditions $(2.2 d)$ are replaced by $\partial F / \partial T-(T-\tilde{c}) \tilde{\mathscr{F}}^{-1} \partial F / \partial X+\sigma_{1}(T-\tilde{c}) \hat{\mathscr{G}}^{-1}$ and $\mathrm{P}$ $-\kappa_{2} X \hat{\mathscr{F}}^{-1}$, respectively. The extra inertial effect in the kinematic condition is again due to the enhanced $u_{1}^{*}$ in the incident motion. In the air motion the only extra change is that $\hat{u}$ tends to $-(T-\tilde{c}) \tilde{\mathscr{F}}^{-1}$ at $\hat{y}=F$, a condition which provokes an additional uniform shearing within the air gap. Altogether the extra contributions to the velocities $u^{*}, v^{*}$ and pressure $p^{*}$ influence the shape-pressure interactions in such a way that the equations generalize from $(2.3),(2.5)$ to

$$
\begin{gathered}
{\left[\partial_{T}-(T-\tilde{c}) \tilde{\mathscr{F}}^{-1} \partial_{X}\right]^{2} F+\sigma_{1} \hat{\mathscr{G}}^{-1}=\frac{1}{\pi} f_{-\infty}^{\infty}\left[P_{Q}(Q, T)-\kappa_{2} \hat{\mathscr{F}}^{-1}\right] \frac{\mathrm{d} Q}{X-Q},} \\
\partial_{X}\left[F^{3} \frac{\partial P}{\partial X}\right]=12 \Gamma\left[\frac{\partial F}{\partial T}-\frac{1}{2}(T-\tilde{c}) \tilde{\mathscr{F}}^{-1} \frac{\partial F}{\partial X}\right] .
\end{gathered}
$$


The constant $\tilde{c}$ can be absorbed into the scaled time $T$, but because of the factor $1 / 2$ in $(2.9 b)$ the two $(T-\tilde{c})$ terms above cannot both be absorbed into a moving spatial coordinate, a feature which indicates the non-trivial influence of the $\tilde{\mathscr{F}}$ factor.

If, instead of the balance in $(2.4 b)$, viscous forces are negligible then in the air gap $\rho_{2}\left(\partial / \partial t^{*}+u^{*} \partial / \partial x^{*}+v^{*} \partial / \partial y^{*}\right) u^{*}$ would be expected to counteract $-\partial p^{*} / \partial x^{*}$, imposing the ordering $\rho_{2} V^{2} /\left(\delta^{2} \ell\right) \sim \rho_{1} V^{2} /(\delta \ell)$ along with the latter being much greater than $\mu_{2} V /\left(\ell^{2} \delta^{3}\right)$ and $\rho_{2} g$. A flow with zero vorticity has the leading term in $u^{*} \propto U(X, T)(V / \delta)$, giving $v^{*} \propto-y^{*} U_{X} V /(\delta \ell)$ through the continuity equation. This yields two equations from momentum and from interface kinematics $\left(\partial f / \partial t^{*}=\right.$ $\left.v^{*}-u^{*} \partial f / \partial x^{*}\right)$,

$$
U_{T}+U U_{X}=-P_{X}, \quad F_{T}+(U F)_{X}=0,
$$

replacing (2.5). Equations $(2.10 a, b)$ with (2.3) for $U, P, F$ agree with Wilson's (1991) and correspond to the range $\delta \sim \rho_{2} / \rho_{1}, r e_{1} \gg \mu_{2} /\left(\mu_{1} \delta^{2}\right)$ together with $(2.7 d)$. It is interesting that the purely inviscid account relies on the gap (of relative width $\delta$ ) being much thinner than in the lubrication/inviscid account of (2.7a), as well as on local water Reynolds numbers larger than that in (2.6). The gap in the inviscid case here is thinner essentially because a smaller $\delta$ tends to increase the typical $\left|u_{2}^{*}\right|$ (which is of order $V / \delta$ from continuity) and so raises the local air Reynolds number $\left|u_{2}^{*}\right| \ell / \nu_{2}$ based on $\left|u_{2}^{*}\right|$, thus accentuating inertial forces compared with viscous forces.

If the wall is moving in its plane there is no change to (2.3), (2.5) unless the wall velocity has $u^{*}$ of order $V / \delta$, in which case $\left(\partial\left(U_{W} F\right) / 2\right) / \partial X$ is added to $\partial F / \partial T$ in (2.5). Here $U_{W}$ is the scaled wall velocity, cf. the effect in (2.9b). Surface tension effects can also be incorporated in principle, for example see McKinley \& Wilson (2001) and others mentioned in $\S 1$, but we will confine our attention to solutions of the basic cases above.

\section{The numerical range of the parameters and validity}

It is of interest to examine the assumptions of the theory numerically, to see if the theory holds only for extremely short length scales and/or low approach velocities. Suppose the fraction $\delta$ is $1 / 100$, thus satisfying the requirements $(2.7 a, b)$ reasonably well. Then from (2.6) the typical local Reynolds number $r e_{1}$ is $1 /\left(55 \delta^{2}\right)$, or about 200 , for the present balances to apply, and the corresponding global value $\operatorname{Re}_{1}\left(\approx r e_{1} / \delta\right)$ is about $2 \times 10^{4}$. Hence the product $V r$ is approximately $200 \mathrm{~cm}^{2} \mathrm{~s}^{-1}$, since $v_{1} \approx 0.01 \mathrm{~cm}^{2} \mathrm{~s}^{-1}$. The theory thus holds for a body of water with typically a $1 \mathrm{~cm}$ radius of curvature approaching a solid surface at normal speed $2 \mathrm{~m} \mathrm{~s}^{-1}$ or a $1 \mathrm{~mm}$ radius of curvature at speed $20 \mathrm{~m} \mathrm{~s}^{-1}$. If $\delta$ is instead $1 / 20$, say, then these speeds read 0.017 and $0.17 \mathrm{~m} \mathrm{~s}^{-1}$, respectively. The range covered in the theory is thus quite wide. The local Froude numbers in the 1/100 case above are over 4000, thus complying with $(2.7 c, d)$ well, and similarly for the $1 / 20$ case. The typical air speeds induced are of the order $V / \delta$, on the other hand, from $\S 2$, thus spanning a range from 0.033 to $2 \times 10^{3} \mathrm{~m} \mathrm{~s}^{-1}$ according to the above estimates, while the local pressure $p^{*}$ is of order $\rho_{1} V^{2} / \delta$ which also covers a wide numerical range. (In some configurations, compressibility is also likely to become a significant factor, see $\S 6$.)

Further, a diagram of the $\left(r e_{1}, \delta\right)$ plane as presented in figure $2(a)$ demonstrates the implications of the inviscid inertial order $\delta \sim \rho_{2} / \rho_{1}$ and the viscous lubrication order $r e_{1} \sim \mu_{2} /\left(\mu_{1} \delta^{2}\right)$. The crossover between the two occurs for a critical $r e_{1}$ range of order $v_{2} \rho_{1} /\left(v_{1} \rho_{2}\right)$. The numbers shown in the diagram are based on the air-water ratios of density and viscosity and on replacing an equal ordering by exact equality, 

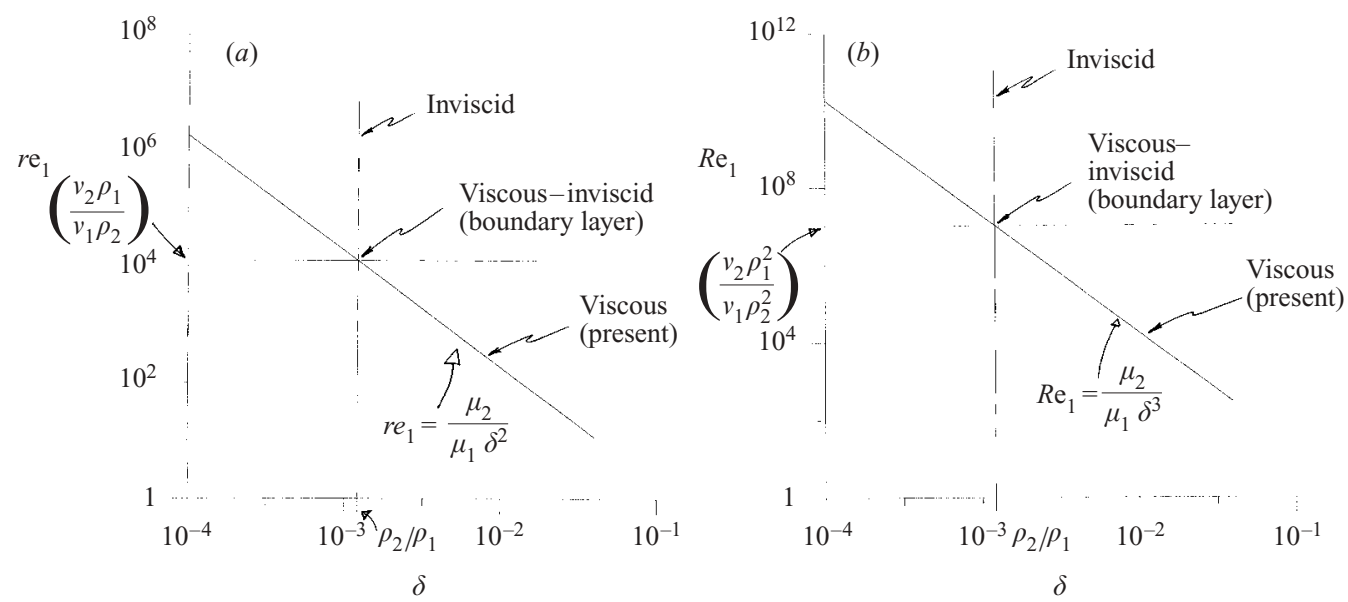

FIGURE 2. Showing the range of application of the present theory, in terms of the reduced and global Reynolds numbers $r e_{1}, R e_{1}$ in $(a),(b)$ and relative gap width $\delta$.

for demonstration purposes. It follows from the above that, as the gap width $\delta$ is decreased (from a suitably large value), with a fixed $r e_{1}$ say well above critical the inviscid-flow description of cushioning in $(2.10 a, b)$ is encountered first whereas $r e_{1}$ below critical leads to the viscous-flow description of cushioning in (2.5) acting first, while the critical range brings in the viscous-inviscid, i.e. boundary layer, equations which cover both cushionings. Here the critical $r e_{1}$ is about $1.24 \times 10^{4}$ for water with an air cushion. (This corresponds to a length scale of $1 \mathrm{~mm}$ with approach speed $10 \mathrm{~m} \mathrm{~s}^{-1}$, for example). The lubrication case therefore seems not unrealistic for a wide variety of configurations, i.e. for $r e_{1}$ below about $1.24 \times 10^{4}$.

A diagram similar to that given in figure $2(b)$ also holds for the global Reynolds number $\operatorname{Re}_{1}\left(\sim r e_{1} / \delta\right)$, in the case of an approaching interface which starts in the shape of a parabola. This gives a very high critical $R e_{1}$ around $v_{2} \rho_{1}^{2} /\left(v_{1} \rho_{2}^{2}\right)=1.24 \times 10^{4} \times 828$ which is more than $10^{7}$, as the figure shows, thus again allowing a wide range of applicability for the current mixed inviscid/lubrication theory.

\section{Application to a plunging part-submerged body flow}

This specific configuration which leads to air cushioning arose from Li et al.'s (2001) work on water motion with a free surface induced near a downward moving solid surface. We consider that context in $\S 4.1$ below, followed by an order-of-magnitude reasoning for air effects in $\S 4.2$ and detailed air-water interaction in $\S 4.3$. See also the Li et al.'s results in figure $1(b)$.

\subsection{The physical context}

Li et al. address the fluid motion, with a free surface but without air cushioning, due to a partly submerged vertical flat plate moved in its own plane. The plate is initially at rest with a submerged length $L$ in still water, at time zero. The plate then moves vertically downward with speed $U$. The velocities $u, v$, time $t$, lengths $x, y$ and pressure $p$ will be measured globally here, in terms of $U, L / U, L, \rho_{1} U^{2}$, respectively (e.g. $u^{*}=U u$ ), and the global Reynolds number $R e_{1} \equiv U L / v_{1}$ in this context is large. The Cartesian system is here defined so that the $x$-axis points vertically upward along the plate and the $y$-axis horizontally into the water. Then the $x$-component of the 
Navier-Stokes equations for the water motion applies in the non-dimensional form

$$
\frac{\partial u}{\partial t}+u \frac{\partial u}{\partial x}+v \frac{\partial u}{\partial y}=-\frac{\partial p}{\partial x}-(\mathscr{F} r)^{-1}+R e_{1}^{-1} \nabla^{2} u,
$$

in this setting, and similarly for the $y$-component but without the gravity effect. The Froude number here is $\mathscr{F} r \equiv U^{2} /(g L)$. The air motion has

$$
\frac{\partial u}{\partial t}+u \frac{\partial u}{\partial x}+v \frac{\partial u}{\partial y}=-\left(\frac{\rho_{1}}{\rho_{2}}\right) \frac{\partial p}{\partial x}-(\mathscr{F} r)^{-1}+\left(\frac{\nu_{2}}{v_{1}}\right) R e_{1}^{-1} \nabla^{2} u
$$

for its $x$-momentum balance, and similarly for $y$-momentum. The initial horizontal upper surface of the water is at $x=0$, and by symmetry only the region $y \geqslant 0$ needs to be considered.

The main nonlinear motion is in the thin boundary layer (wetted plate) and its wake (dry plate), the latter adjoining a trailing free surface, moving downwards close to the plate and of thickness $O(\epsilon)$ where $\epsilon$ denotes $R e_{1}^{-1 / 2}$, from (4.1a). The contact line between wet and dry plate is assumed not to slip relative to the plate. The boundary layer where $-1-t<x<-t$ has vertical velocity and coordinate $u, x$ of order unity, time of order unity and the viscous scaling $y=\epsilon Y$ with $v$ of order $\epsilon$, while the pressure gradient $\partial p / \partial x$ is independent of $Y$ and so is cancelled out by the gravity force to match horizontally with the bulk of the water flow outside. In the wake where $-t<x<0$ above the boundary layer, the same scalings hold but $p$ is zero (atmospheric pressure), with the side free surface lying within an unknown horizontal distance $O(\epsilon)$ (say $\epsilon f_{2}(x, t)$ ) of the plate for $x$ of order unity and requiring $\partial u / \partial Y=0$ and $\tilde{v}-\partial f_{2} / \partial t-u \partial f_{2} / \partial x=0$ at $Y=f_{2}(x, t)$, where $v=\epsilon \tilde{v}$. Large Froude number $\mathscr{F} r$ of order $\epsilon^{-1}$ at least is assumed here. Use of the Prandtl transposition based on $Y-f_{2}$ (i.e. working in terms of $\tilde{v}-\partial f_{2} / \partial t-u \partial f_{2} / \partial x$ and $Y-f_{2}$ instead of $\tilde{v}$ and $Y$ in turn) and coordinates moving downward with speed unity then shows (Papadopoulos 2000; Li et al. 2001, 2002) that the above nonlinear motion is equivalent to the unsteady boundary layer and wake of an aligned flat plate started impulsively from rest in unbounded fluid. These regions yield a small $O(\epsilon)$ unsteady displacement which drives the rest, the bulk, of the water flow.

The bulk where $x, y$ are $O(1)$ remains nearly still, with $u, v, p$ all being of order $\epsilon$. Thus from (4.1a) potential flow properties apply in a quarter plane, subject to mixed boundary conditions of effectively zero pressure at the unknown upper and side (dry plate) free surfaces and at infinity and of prescribed normal pressure derivative at the wetted plate. The flow solution there ( $\mathrm{Li}$ et al.), corresponding to the large Froude number of order $\epsilon^{-1}$, determines the scaled upper and side $\left(f_{2}\right)$ free-surface shapes. It is found in particular that in the assumed dry-plate range, $f_{2}$ is indeed positive at sufficiently small positive times, but impingement, in the sense of $f_{2}$ approaching $0+$, occurs eventually at some time $t=t_{0}$ and position $x=x_{0}$ within $-t_{0}<x_{0}<0$. The impingement time $t_{0}$ is generally of order unity although it decreases monotonically with decreasing Froude number and also depends slightly on the precise plate conditions. The response

$$
f_{2} \sim a_{2}\left(x-x_{0}\right)^{2}+b_{2}\left(t_{0}-t\right),
$$

near $x=x_{0}$ as $t \rightarrow t_{0}-$, with $a_{2}, b_{2}$ being positive constants, describes the local behaviour as impact onto the plate is approached. 
4.2. Orders of magnitude for air effects

Air dynamics can be expected to come into play only locally, around time $t_{0}$. Usually this is taken to be an extra inviscid effect (see references in $\S 1$ ), but in the present context the air gap is thin throughout, of order $\epsilon$ at most, lying between the descending plate and the moving side free surface, both of which shear the air in the gap. Moreover the air gap becomes thinner at the onset of impact and so its effect is viscous. The reason is that, given the velocity and length factors $U$ and $L$, the global air Reynolds number $R e_{2}=U L / \nu_{2}$ is a fraction $v_{1} / \nu_{2}(\approx 1 / 15)$ of that of water $R e_{1}$, implying that a typical estimated boundary-layer thickness of air exceeds that of water by a factor $\left(v_{2} / v_{1}\right)^{1 / 2} \approx 4$, from the scales simplified by $(4.1 a, b)$. Hence the $O(\epsilon)$ air gap above is, if anything, thin relative to an air boundary-layer scale and must be governed by a viscous lubrication balance, the 'thin' limit of the boundary-layer balance, and this difference becomes accentuated as the gap narrows near time $t_{0}$.

Prior to any cushioning, the water boundary layer and wake dynamics dominate over the thin air flow effects because of the smallness of $\rho_{2} / \rho_{1}, \mu_{2} / \mu_{1}$, in a manner similar to that described at the start of $\S 2$, yielding here the free-surface conditions on the water given in $\S 4.1$. In addition, however, the slower bulk of the water flow in $\S 4.1$ is also unaffected earlier on, provided that $\epsilon$ is much greater than $\mu_{2} / \mu_{1}$ in view of the pressure gradients of typical order $\mu_{2} / \mu_{1}$ generated in the air gap from $(4.1 b)$ and those of order $\epsilon$ in the water bulk. (Otherwise, an early interaction occurs; see $\S 6)$. The water Reynolds number $R e_{1}$ is thus assumed here to be significantly less than $\left(\mu_{1} / \mu_{2}\right)^{2} \approx 3000$. The air flow of order- $\epsilon$ thickness, then, is forced by the prescribed $u$ (wake) and $v$ (kinematic) values from the water flow solution at the known interface $\epsilon f_{2}$, as well as by the $u, v$ values imposed by the moving plate, and is governed by the boundary-layer balance with unknown pressure $p$ if $v_{1} \sim v_{2}$, or merely by the lubrication balance (giving $p$ more explicitly) owing to the numerical smallness of $v_{1} / v_{2}$. The air gap solution determines the air pressure variation and $\mu_{2} \partial u_{2} / \partial y$ at the interface and hence a small correction to the water flow solution.

The air lubrication balance is found to prevail at the onset of impact in (4.2), producing high pressure gradients which lead to air cushioning locally. An estimate from the air dynamics above gives $v$ of order $\epsilon$ from the kinematic condition, so that $u \sim\left|x-x_{0}\right| / f_{2}$ from mass conservation while the vertical pressure gradient $\partial p / \partial x \sim\left(\mu_{2} / \mu_{1}\right) u / f_{2}^{2}$ from the viscous forces in the air, which overwhelm the inertial ones because of $(4.1 b)$. This pressure gradient, acting on the water bulk where $x, y$ are comparable, induces a $v$-component of order $\left|t-t_{0}\right|\left(\mu_{2} / \mu_{1}\right)\left|x-x_{0}\right| / f_{2}^{3}$ from the balance of $\partial v / \partial t$ against the pressure gradient, using (4.1a). As impact approaches in (4.2) the induced $v$-component is thus growing in size as $\left|t-t_{0}\right|^{-3 / 2}\left(\mu_{2} / \mu_{1}\right)$, since locally $\left(x-x_{0}\right)^{2}$ and the short scaled time $\left|t-t_{0}\right|$ are comparable and $f_{2} \sim\left|t-t_{0}\right|$. This size becomes comparable with the original kinematic one of order $\epsilon$ when

$$
t-t_{0} \sim\left(\frac{\mu_{2}}{\epsilon \mu_{1}}\right)^{2 / 3},
$$

which is small and defines the local air-cushioning time scale. Here we set $\Delta=\rho_{2} /\left(\epsilon \rho_{1}\right)$, which is of order $\mu_{2} /\left(\epsilon \mu_{1}\right)$ since $v_{1} \sim \nu_{2}$, leaving (4.3) as order $\Delta^{2 / 3}$, for convenience. So the induced pressure gradients are predicted to be of order $\epsilon \Delta^{-2 / 3}$, in view of (4.3).

\subsection{Details in the three regions}

In view of the order-of-magnitude estimates in $\S 4.2$ we expect $x-x_{0}, t-t_{0}$ to be of the form $\Delta^{1 / 3} X, \Delta^{2 / 3} T$, respectively, with $X, T$ of order unity, and we also expect 
three distinct $y$-scales of orders $\epsilon \Delta^{2 / 3}, \epsilon, \Delta^{1 / 3}$ to operate. The $y$-scales define regions denoted as I-III, respectively. The air gap width is $\epsilon \Delta^{2 / 3} F(X, T)$ with $F$ of order one but unknown.

The thin air gap I now has $y=\epsilon \Delta^{2 / 3} \hat{\hat{y}}$ with $0 \leqslant \hat{\hat{y}} \leqslant F$ of order unity and to a first approximation

$$
[u, v, p]=\left[\Delta^{-1 / 3} \hat{\hat{u}}, \epsilon \hat{\hat{v}}, \epsilon \Delta^{-1 / 3} \hat{\hat{p}}\right] .
$$

Hence the balances of mass and momentum yield, with $\bar{\Gamma}$ standing for $\nu_{2} / v_{1}$ which is of the same order as $\Gamma$,

$$
\hat{\hat{u}}_{X}+\hat{\hat{v}}_{\hat{y}}=0, \quad 0=-P_{X}(X, T)+\bar{\Gamma} \hat{\hat{u}}_{\hat{y} \hat{y}}
$$

from (4.1b), since $\Delta, \epsilon$ are small, with $\hat{\hat{p}}=P(X, T)$ being independent of $\hat{\hat{y}}$ from the lateral momentum equation. The boundary conditions require

$$
\begin{aligned}
& \hat{\hat{u}}=\hat{\hat{v}}=0 \quad \text { at } \quad \hat{y}=0, \\
& \hat{\hat{u}}=0, \quad \hat{\hat{v}}=F_{T} \quad \text { at } \quad \hat{y}=F(X, T),
\end{aligned}
$$

where the no-slip constraint (4.5c) holds because the air-gap velocities $u$ in (4.4) are much greater than the typical wall velocity of unity. The condition on $\hat{\hat{u}}$ in $(4.5 d)$ is necessary as in $\S 2.1$, for continuity of the present large velocities $u\left(\sim \Delta^{-1 / 3}\right)$ with the smaller velocities induced in the water at the interface with region II $(u \sim 1$, see (4.6) below), while the condition on $\hat{v}$ is the kinematic one but with the contribution from $u \partial f / \partial x$ negligible owing to the condition on $\hat{\hat{u}}$ just described. The contribution from $\partial f / \partial t$ to the kinematic condition is of order $\epsilon$ here, as it was in $\S 4.1$. Also here, continuity of the tangential stress at the interface is accommodated by the shear layer II which is described in the next paragraph. The solution of (4.5a-d) leads to (2.5), with $\bar{\Gamma}$ replacing $\Gamma$, a relationship between the interface shape and pressure.

The neighbouring water region II (a shear layer) is also thin, with $y=\epsilon Y$ of order $\epsilon$. Here the flow response involves a small disturbance from the local wake velocity profile $u_{0}(Y)$ say, produced by the earlier evolution as in $\S 4.1$, such that

$$
[u, v, p]=\left[u_{0}(Y)+\Delta^{1 / 3} u_{1}, \epsilon v_{1}, \epsilon \Delta^{-1 / 3} P\right]
$$

at leading order. The sheared profile $u_{0}$ is smooth and satisfies $u_{0}^{\prime}(0)=u_{0}(\infty)=0, u_{0}(0)$ and $u_{0}^{\prime \prime}(0)$ are $O(1)$ non-zero constants in general, while the associated stream function profile $\psi_{0}(Y)$ has the properties $u_{0}=\psi_{0}^{\prime}, \psi_{0}(0)=0$, and $\psi_{0}(\infty)=-\delta_{\infty}$ is an $O(1)$ constant representing the local scaled wake thickness. The fact that $\delta_{\infty}$, like $u_{0}$ and $\psi_{0}$, is independent of the present relatively fast time scale is again due to it being a result from the earlier slower-scale evolution in $t\left(\approx t_{0}\right.$ here). The pressure in (4.6), which matches that in (4.4) across the interface at $Y=0+, \hat{\hat{y}}=F$, is independent of $Y$ from the lateral momentum again, but plays negligible part in region II. Owing to (4.1a), the $x$ momentum balance merely leaves $\partial u_{1} / \partial T$ having to be zero, provided that $\rho_{2} / \rho_{1}$ greatly exceeds $\epsilon^{4}$, and continuity requires $\partial u_{1} / \partial X+\partial v_{1} / \partial Y$ to be zero. The relevant boundary conditions are

$$
\begin{gathered}
u_{1 Y}=0, \quad v_{1}=F_{T} \quad \text { at } Y=0+, \\
u_{1} \propto X \text { as }|X| \rightarrow \infty .
\end{gathered}
$$

Here (4.7a) follows from the largeness of the typical $\mu_{1} \partial u / \partial y$ contribution in II, of order $\mu_{1} \Delta^{1 / 3} / \epsilon$, relative to the value $\mu_{2} \partial u / \partial y \sim \mu_{2} \Delta^{-1} / \epsilon$ in I at the interface, and 
from the kinematic condition; the contributions from $u_{0}, \partial u_{0} / \partial Y$ are too small at the interface to affect $(4.7 a)$. This relative largeness holds provided that $\rho_{2} / \rho_{1} \gg \epsilon^{4}$ again. The condition (4.7b) stems from the earlier wake evolution and its $x$ variation, producing an $O\left(\Delta^{1 / 3}\right)$ correction from $u_{0}$. An acceptable flow solution is found to be $u_{1}=X u_{10}(Y), v_{1}=-\psi_{10}(Y)+G(X, T)$. Here the effective correction profile $u_{10}(Y)$ is arbitrary, but subject to $u_{10}^{\prime}(0), u_{10}(\infty)$ being zero, while $\psi_{10}^{\prime}(Y)=u_{10}(Y)$ with $\psi_{10}(0)$ being zero and $\psi_{10}(\infty)$ is generally non-zero. The unknown function $G$ representing local effects is $\partial F / \partial T$ from the interface requirement (4.7a). Hence at large positive $Y$ values

$$
v_{1} \sim-\psi_{10}(\infty)+F_{T}, \quad u_{1} \rightarrow 0,
$$

in readiness for matching with region III's flow solution.

Region III is where the horizontal and vertical lengths are comparable, so that $y=\Delta^{1 / 3} \bar{y}$ with $\bar{y}$ of order one, and the above flow behaviour points to the form

$$
[u, v, p]=\left[\epsilon \bar{u}_{1}, \epsilon \bar{v}_{1}, \epsilon \Delta^{-1 / 3} \bar{p}_{1}\right]
$$

and similarly for the corresponding stream function. The induced pressure size exceeds the earlier pressure size $\epsilon$ of $\S 4.1$ and the Froude number plays no part. From (4.1a), the mass and momentum balances reduce to $\partial \bar{u}_{1} / \partial X+\partial \bar{v}_{1} / \partial \bar{y}, \partial \bar{u}_{1} / \partial T+$ $\partial \bar{p}_{1} / \partial X, \partial \bar{v}_{1} / \partial T+\partial \bar{p}_{1} / \partial \bar{y}$ all being zero, since $\epsilon, \Delta$ are small, and so potential flow applies. The boundary conditions require a match to II, implying that $\bar{p}_{1}$ tends to $P$ and $\bar{v}_{1}$ to $v_{1}$ in (4.8) as $\bar{y} \rightarrow 0+$, along with boundedness far away. Therefore $\bar{p}_{1}-\mathrm{i} \partial \bar{\psi}_{1} / \partial T$ is analytic in $X+\mathrm{i} \bar{y}$, leading to the relation (2.3), since $\psi_{0}(\infty)$ is constant.

Hence $F, P$ are again controlled by the coupled nonlinear system (2.3), (2.5), subject mainly to $P \rightarrow 0$ at large $|X|$ and to consistency with (4.2) at early times. The justification of the inviscid-lubrication interplay between I, III, along with the passive role of the shear layer II in-between, supports the wider applications considered throughout $\S 2$.

\section{Solution properties for $F, P$}

\subsection{Computational method and results}

The solution of (2.3), (2.5) for $F, P$ is addressed first here. Time-marching computations were performed by means of a fourth-order compact difference scheme.

Variables $(q, r, s, E) \equiv\left(\partial F / \partial T, \partial P / \partial X, \partial^{2} P / \partial X^{2}, \partial F / \partial X\right)$ were treated as unknowns in addition to $F, P$, leaving (2.5) as a nonlinear equation between $F, s, E, r, q$ and (2.3) as $\pi \partial q / \partial T=I$. The principal-value integral $I$ was expressed as a summation of fourth-order accuracy using discretized values of $r, s$. The resulting six first-or zero-order partial differential equations were written in fourth-order accurate compact difference form spatially and solved iteratively at each time step, subject to the relevant boundary conditions at endpoints representing $\pm \infty$. Time differencing was of second-order accuracy. Thus (2.3), (2.5), respectively, were discretized at each $X=X_{i}$ station as

$$
\begin{gathered}
\pi\left(q_{i}^{(m+1)}-q_{i}^{(m)}\right) / \Delta T=\tilde{\alpha} r_{i}+\tilde{\beta} s_{i}+\tilde{\gamma}, \\
F_{i}^{3} s_{i}+3 F_{i}^{2} E_{i} r_{i}=12 q_{i},
\end{gathered}
$$


with uniform spatial and temporal step sizes $\Delta X, \Delta T$, combined with

$$
\begin{gathered}
q_{i}=\left(F_{i}^{(m+1)}-F_{i}^{(m)}\right) /(\Delta T), \\
g_{1} r_{i-1}+g_{2} r_{i}+g_{3} r_{i+1}=\left(\tilde{g}_{1} P_{i-1}+\tilde{g}_{2} P_{i}+\tilde{g}_{3} P_{i+1}\right) /(\Delta X), \\
\delta_{1} s_{i-1}+\delta_{2} s_{i}+\delta_{3} s_{i+1}=\left(\tilde{\delta}_{1} P_{i-1}+\tilde{\delta}_{2} P_{i}+\tilde{\delta}_{3} P_{i+1}\right) /(\Delta X)^{2}, \\
\left(g_{1} E_{i-1}+g_{2} E_{i}+g_{3} E_{i+1}\right)=\left(\tilde{g}_{1} F_{i-1}+\tilde{g}_{2} F_{i}+\tilde{g}_{3} F_{i+1}\right) /(\Delta X) .
\end{gathered}
$$

In the above, the superscripts $(m),(m+1)$ refer to the known values at time $T$ and the unknowns at $T+\Delta T$, in turn, while the average

$$
F_{i} \equiv \frac{1}{2}\left(F_{i}^{(m)}+F_{i}^{(m+1)}\right)
$$

gives second-order accuracy at time $T+\Delta T / 2$ and similarly for all the other quantities. So $F_{i}^{(m+1)}$ in (5.3) can be replaced by $2 F_{i}-F_{i}^{(m)}$ and likewise for $q_{i}^{(m+1)}$ in (5.1). Also the subscript $i$ denotes the values at $X=X_{i}$ and the fourth-order coefficients are given by

$$
\begin{aligned}
& \left(g_{1}, g_{2}, g_{3}\right)=\left(\frac{1}{3}, \frac{4}{3}, \frac{1}{3}\right), \\
& \left(\tilde{g}_{1}, \tilde{g}_{2}, \tilde{g}_{3}\right)=(-1,0,1), \\
& \left(\delta_{1}, \delta_{2}, \delta_{3}\right)=\left(\frac{1}{12}, \frac{5}{6}, \frac{1}{12}\right), \\
& \left(\tilde{\delta}_{1}, \tilde{\delta}_{2}, \tilde{\delta}_{3}\right)=(1,-2,1),
\end{aligned}
$$

while in (5.1) the coefficients are

$$
\begin{gathered}
\tilde{\alpha}=\phi_{i i}-\tilde{\phi}_{i i}+\left(\tilde{\phi}_{i i-1}+\tilde{\phi}_{i i+1}\right) / 2 \\
\tilde{\beta}=(\Delta X)\left[\bar{\phi}_{i i}+\left\{\hat{\phi}_{i i-1}-2 \hat{\phi}_{i i}+\hat{\phi}_{i i+1}\right\} / 6\right] \\
\tilde{\gamma}=\sum_{j=0}^{N}\left\{\phi_{i j} r_{j}+(\Delta X) \bar{\phi}_{i j} s_{j}+\tilde{\phi}_{i j}\left(r_{j+1}-2 r_{j}+r_{j-1}\right) / 2\right. \\
\left.+(\Delta X) \hat{\phi}_{i j}\left(s_{j+1}-2 s_{j}+s_{j-1}\right) / 6\right\}-\tilde{\alpha} r_{i}-\tilde{\beta} s_{i} .
\end{gathered}
$$

Here

$$
\begin{gathered}
\phi_{i j}=\ln \left|\left(i-j+\frac{1}{2}\right) /\left(i-j-\frac{1}{2}\right)\right|, \\
\bar{\phi}_{i j}=(i-j) \phi_{i j}-1, \\
\tilde{\phi}_{i j}=(i-j) \bar{\phi}_{i j}, \\
\hat{\phi}_{i j}=(i-j) \tilde{\phi}_{i j}-\frac{1}{12},
\end{gathered}
$$

and the range of the subscript $i$ or $j$ is from 0 to $N$. At each $i$, the program developed solves for the average values $r_{i}, E_{i}, s_{i}$ and $q_{i}$ together, $P_{i}, F_{i}$ from (5.4), (5.6), (5.1) and (5.2) together, (5.5), (5.3), in that order, with all other quantities at $i-1, i, i+1$ taking their latest known values within each equation. The procedure is swept through the domain from a suitably large negative $X_{i}=X_{1}$ to a suitably large positive $X_{i}=X_{N-1}$ value with the boundary conditions being imposed directly on the six functions above at $X_{0}$ and $X_{N}$, respectively. This sweeping procedure is repeated until successive iterates are sufficiently close in value, with an iterative tolerance of $10^{-6}$ in all $P_{i}$ values usually being imposed. Tests were applied to this tolerance, to the starting time and to the $X$-grid in order to check on the numerical accuracy, and representative tests are indicated in some of the figures below. 


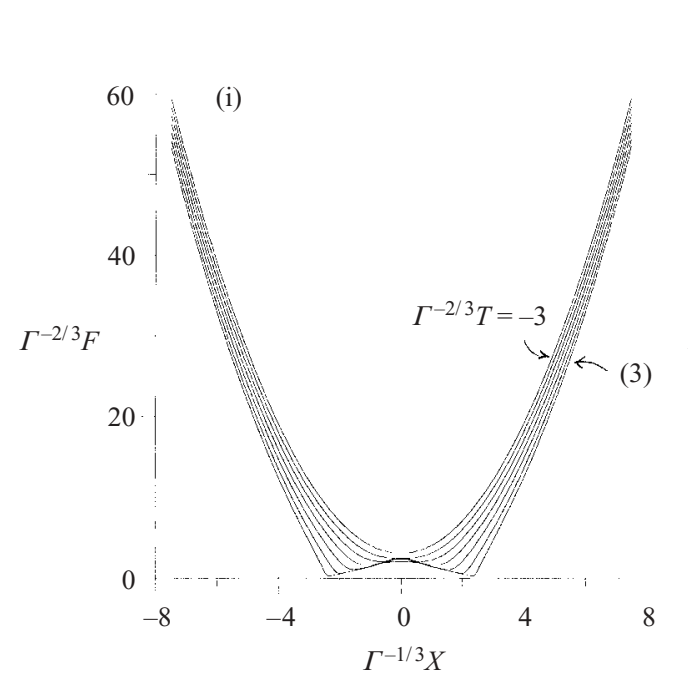

(a)
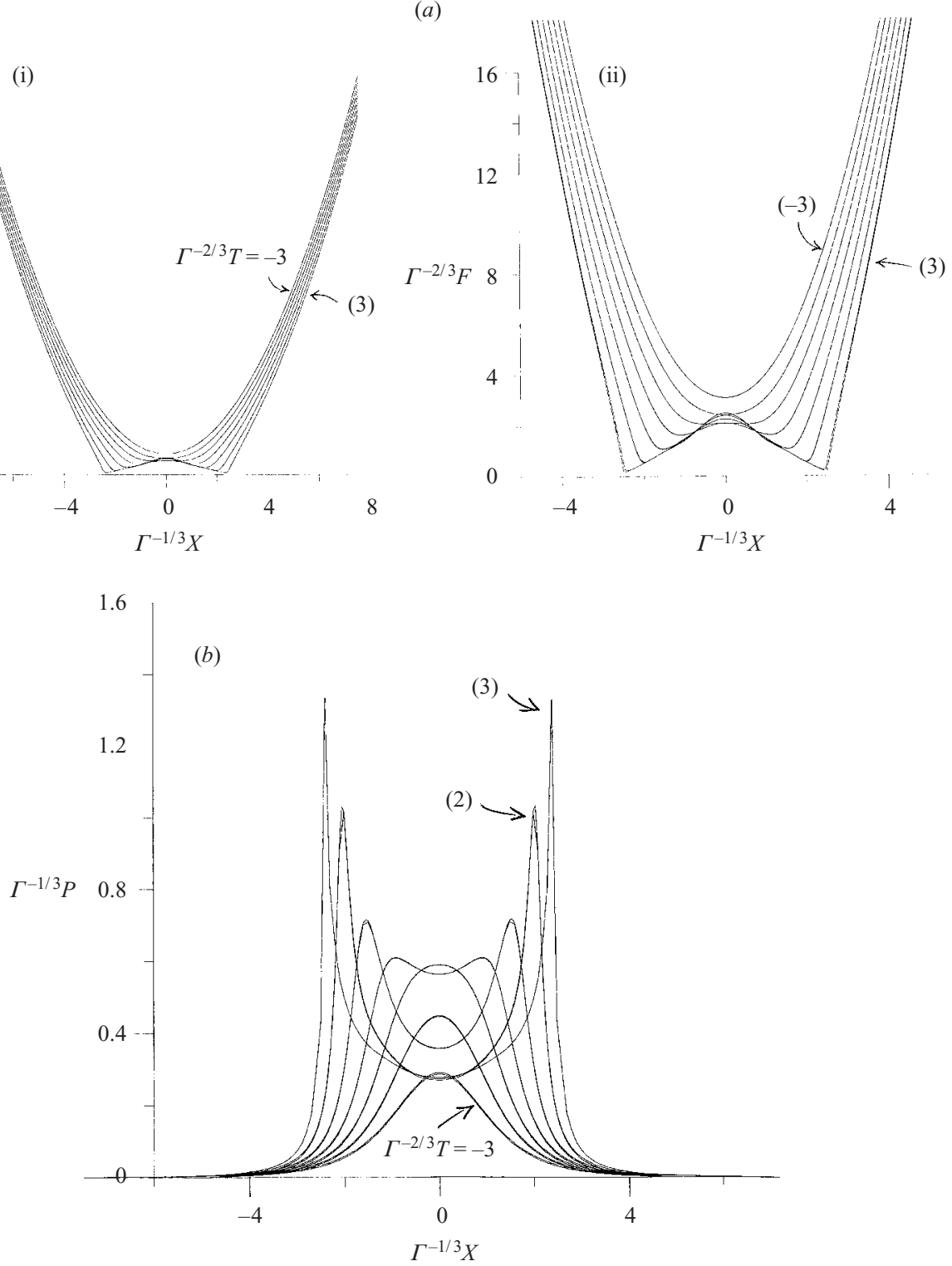

FIGURE 3. Computational solutions of (2.3), (2.5), starting with a parabolic local interface shape. (a) Interface shape $F,(b)$ pressure $P, v s$. $X$ at various times $T$.

Results are presented in figure 3 for the case of $\S 2$ (and $\S 4$ ) with the constants $a_{2}$, $b_{2}$ normalized to unity. Figure 3(a)(i) shows the scaled interface shape $F$ against $X$ at various scaled times $T$, obtained with an $X$ grid distribution of $151 \times 0.1$, time step 0.005 , with a starting time $T$ of -4 . Figure 3(a)(ii) gives corresponding closer-up results from three different grids, one as above, one with the $X, T$ steps halved and one with the same $X$ step but fewer points. The three sets of results are extremely close at most times and only differ slightly at late times. Likewise figure $3(b)$ presents the scaled pressure response from two grids, with $X$ steps of $0.1,0.05$, indicating almost identical features, including the relatively short scaled (spiky) behaviour appearing 

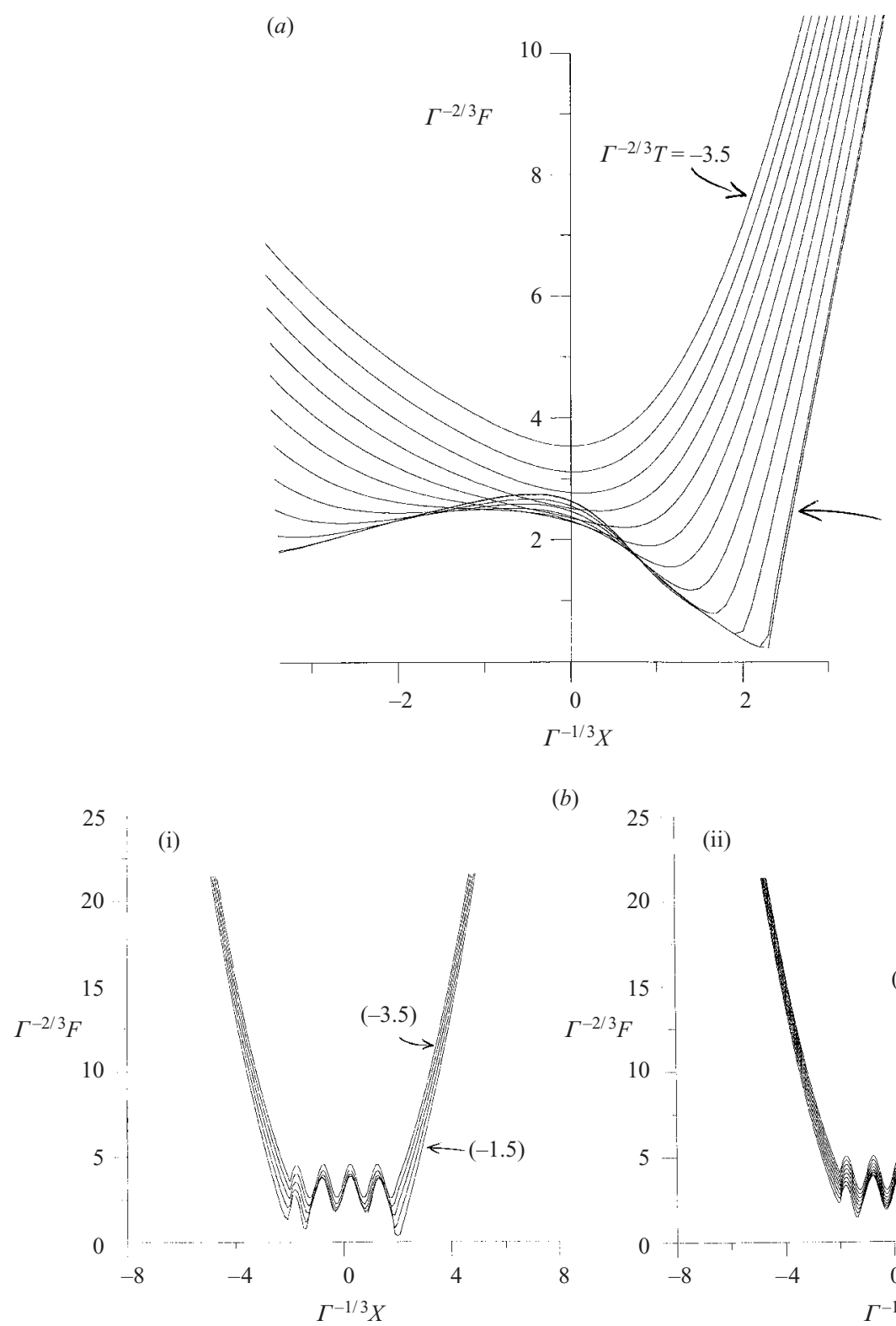

(b) 25

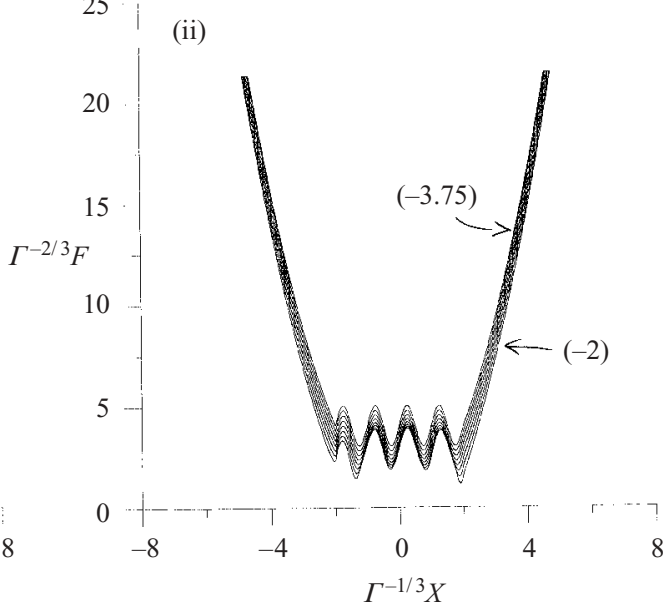

FIGURE $4(a, b)$. For caption see facing page.

towards the final times reached by the computations. Altogether the results show the parabola essentially as in (4.1) or (2.8) with $A=-1, B=X^{2}$, at first, as the coupling is only weak for sufficiently large negative times. Thereafter the coupling gradually grows, followed by a cushioning interaction which decelerates and eventually reverses the approach of the parabola head to the wall, and finally a touching down onto the wall is indicated. The suggested touchdown is accompanied by increased pressures locally.

A simpler computational scheme of only second-order spatial accuracy was also run, for comparison. This indicated the efficiency of the fourth-order scheme. Fourth-order 


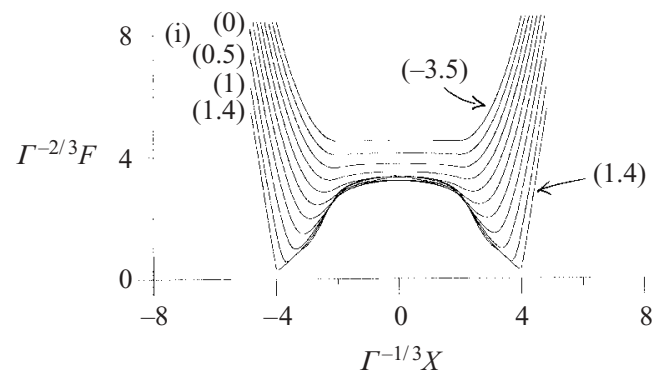

(ii)

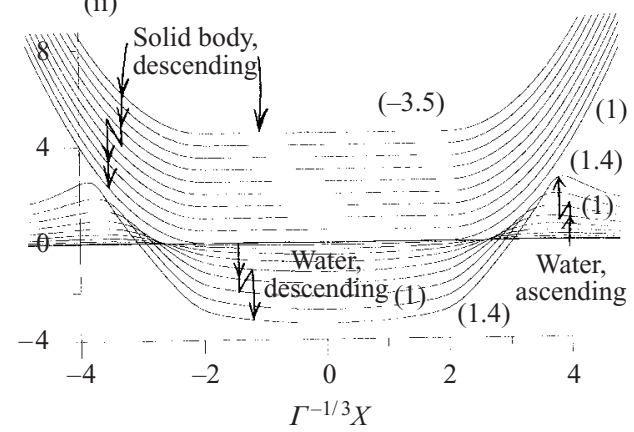

(c)

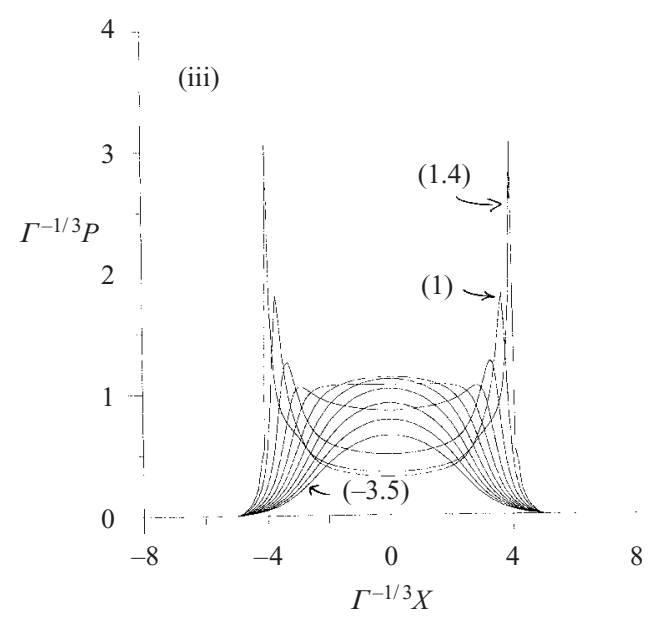

(d)

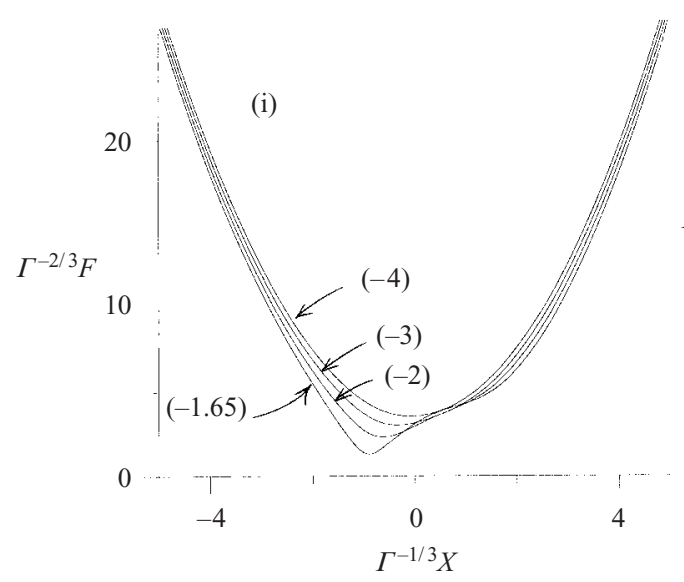

(ii)

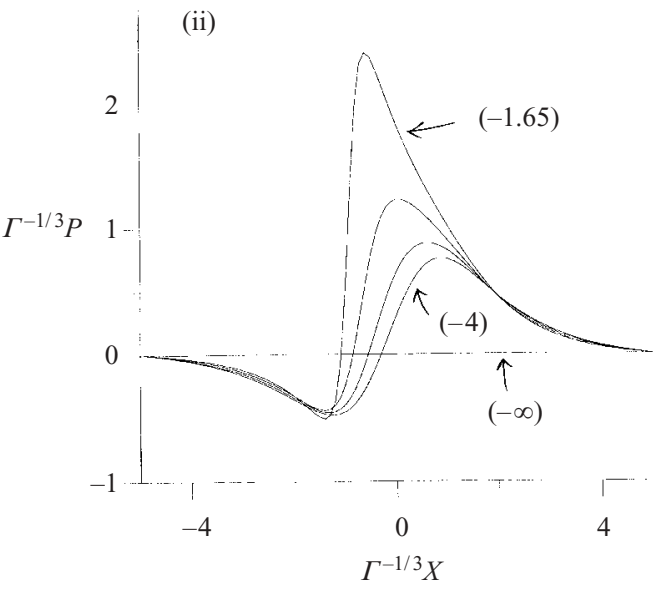

FIGURE 4. Time-marched solutions computed for (2.8) and $(2.9 a, b)$. ( $a$ ) With non-symmetry in the early approaching shape. $(b)$ With corrugation and non-symmetry. $(c)$ With a flat-bottomed shape. (d) With a significant gravity contribution.

computations were performed as well for model problems in which $I / \pi$ in (2.3) was replaced by $\pm r$ or $\pm s$. The former cases correspond in a sense to a supersonic counterpart of the subsonic law (2.3). All cases apparently led to a touchdown in a form similar to that in figure 3. Analysis of simplified models supported the occurrence of the phenomenon.

In view of the generalizations described in $\S 2.2$, we extended the computational study to (2.8) and (2.9) to allow for other incident interface shapes and for reduced Froude numbers. The results are shown in figure 4 . These often lead to first touchdown 
occurring at one position only, as distinct from the double touchdown due to symmetry in figure 3. The results include a case with a flat bottom modelling the shape mentioned in Driscoll \& Lloyd (1982), Nethercote et al. (1986), Howison et al. (1991, 2002), Wilson (1991), and other cases such as wedge-like shapes have also been investigated. Figure $4(a)$ has non-symmetry in the approaching interface shape, such that in (2.8) $A$ is -1 and $B$ is $\left(b_{1}+B_{3}\right) X^{2} /\left(1+B_{3}\right)$ with $b_{1}=1 / 4$ and $B_{3}=\exp (X)$, as the figure indicates. Figure $4(b)$ shows the results for a corrugated shape approaching, with slight non-symmetry. Figure $4(b)(\mathrm{i})$ has an $X$ step of 0.1 , figure $4(b)($ ii) has 0.05 to check on the accuracy, which seems to be assured. The case in figure $4(c)$ is the flat-bottomed one where $A=-1$, but $B$ is $(X+2)^{2}+1$ for $X<-2,1$ for $-2 \leqslant X \leqslant 2$ and $(X-2)^{2}+1$ for $X>2$. The scaled shapes involved are shown not only for an initial air-water interface (2.8) approaching a flat solid surface (or symmetry line), but also for a solid surface, defined as in (2.8) but for all time $T$, moving towards an initially flat water surface. The scaled pressure response remains the same in the two settings. Finally here, figure $4(d)$ includes a gravity contribution as in $(2.9 a, b)$, with $\sigma_{1}, \kappa_{2}, \tilde{c}$ all zero, $\tilde{\mathscr{F}}=\Gamma / 2$, the solution (which includes negative pressures) being presented in the appropriate moving coordinate frame and being non-symmetric because of the gravity effect.

According to all the results whether for an $X$-symmetric or $X$ - non-symmetric interfacial shape, the maximum feedback of pressure from the air cushion at relatively early times occurs near the minimum gap width (the middle), as expected physically. This decelerates the interface there and can even reverse its movement, but the high pressures also force the incident water flow sideways, generating eventually even higher pressures there instead. The latter trend is so powerful that touchdown of the water onto the wall accompanied by very large induced pressures, at one or more sideways positions (thus producing a double peak in the pressure), are indicated within the current scalings. Strictly, the phenomenon is an 'approach to touchdown' as new physics must soon come into the reckoning locally.

\subsection{Touchdown}

The touchdown indicated by all the computational results in figures 3 and 4 suggests in turn examining a finite-time termination of the solution at some station $X_{0}$ and time $T_{0}$, in which a local scale $X-X_{0}=\left(T_{0}-T\right)^{\alpha} \eta$ is assumed to apply. Here $\eta$ is of order unity and the positive power $\alpha$ is unknown. The orders of magnitude imply that

$$
F \sim\left(T_{0}-T\right)^{\alpha / 3+1 / 3} \tilde{F}(\eta), \quad P \sim\left(T_{0}-T\right)^{4 \alpha / 3-5 / 3} \tilde{P}(\eta)
$$

locally, with $\tilde{F}, \tilde{P}$ governed by

$$
\begin{gathered}
\left(\tilde{F}^{3} \tilde{P}^{\prime}\right)^{\prime}=12 \Gamma\left[\alpha \eta \tilde{F}^{\prime}-\frac{1}{3}(\alpha+1) \tilde{F}\right] \\
\alpha^{2} \eta^{2} \tilde{F}^{\prime \prime}+\frac{1}{3} \alpha(\alpha+1) \eta \tilde{F}^{\prime}+\left(\frac{\alpha+1}{9}\right)(\alpha-2) \tilde{F}=\frac{1}{\pi} \int_{-\infty}^{\infty} \frac{\tilde{P}^{\prime}(\hat{\eta}) \mathrm{d} \hat{\eta}}{\eta-\hat{\eta}},
\end{gathered}
$$

from $(2.3),(2.5)$, subject to $\tilde{P}( \pm \infty)=0$. The latter pressure condition is required to match with the (regular) solution away from $\left(X_{0}, T_{0}\right)$, assuming $\alpha<5 / 4$. The solution of the similarity form $(5.10 a, b)$ was sought numerically, but despite many attempts an acceptable solution was not found, at least in a smooth form. The resolution seems to be that the solution of $(5.10 a, b)$ allied with the pressure condition must be irregular at some finite station $\eta=-c_{1}$ say, such that

$$
\tilde{F} \propto\left|\eta+c_{1}\right|^{2 / 3}, \quad \tilde{P} \propto\left|\eta+c_{1}\right|^{-1 / 3} \quad \text { as } \eta \rightarrow-c_{1},
$$


a form consistent with the governing equations locally. Thus a shorter-scale coordinate is necessary for smoothing, say $X-X_{0}=-\left(T_{0}-T\right)^{\alpha} c_{1}+\left(T_{0}-T\right)^{n} \xi$, with $n>\alpha$. This gives with $\xi$ of order unity

$$
F \sim\left(T_{0}-T\right)^{\beta_{1}} \hat{F}(\xi), \quad P \sim\left(T_{0}-T\right)^{\beta_{2}} \hat{P}(\xi)
$$

to leading order, from (5.9), (5.11), where $\beta_{1}=(2 n-\alpha+1) / 3, \beta_{2}=(-n+5 \alpha-5) / 3$. Then $\hat{F}, \hat{P}$ are controlled by

$$
\begin{gathered}
\left(\hat{F}^{3} \hat{P}^{\prime}\right)^{\prime}=-12 \Gamma \alpha c_{1} \hat{F}^{\prime}, \\
\alpha^{2} c_{1}^{2} \hat{F}^{\prime \prime}=\frac{1}{\pi} \int_{-\infty}^{\infty} \frac{\hat{P}^{\prime}(q) \mathrm{d} q}{\xi-q},
\end{gathered}
$$

subject to matching with $(5.11)$ at large $|\xi|$. The prime denotes differentiation in $\xi$ here as opposed to $\eta$ in $(5.10 a, b)$. Integration of $(5.13 a)$ leads to

$$
\hat{F}^{3} \hat{P}^{\prime}=-12 \Gamma \alpha c_{1}(\hat{F}-\hat{B})
$$

leaving $(5.13 b, c)$ as two equations for $\hat{F}, \hat{P}^{\prime}$. The constant $\hat{B}$ must be positive to keep $\hat{F}$ positive. The matching condition can be made more precise however, requiring specifically that

$$
\begin{aligned}
& \hat{F} \sim \lambda_{1} \xi^{2 / 3}, \quad \hat{P} \sim \lambda_{2} \xi^{-1 / 3} \quad \text { as } \xi \rightarrow \infty, \\
& \hat{F} \rightarrow \hat{B}, \quad \hat{P} \sim \lambda_{3}|\xi|^{-1 / 3} \quad \text { as } \quad \xi \rightarrow-\infty .
\end{aligned}
$$

Here $\lambda_{1}=\left(12 \Gamma /\left(\alpha c_{1}\right)\right)^{1 / 3} \lambda_{5}, \quad \lambda_{2}=(2 / 3 \sqrt{3})\left(12 \Gamma \alpha^{5} c_{1}^{5}\right)^{1 / 3} \lambda_{5}, \quad \lambda_{3}=2 \lambda_{2}$ and $\lambda_{5} \equiv$ $3^{5 / 6} / 2^{1 / 3}$. If we try $\hat{F} \sim \lambda_{4}|\xi|^{2 / 3}$ at large negative $\xi$ also with $\lambda_{4}$ positive to keep the scaled gap width positive and of equal order to the far left and right, then the $\hat{B}$ term in $(5.13 c)$ has no leading-order effect in the far field, but although all other terms in $(5.13 b, c)$ balance to determine possible values of $\lambda_{1}$ to $\lambda_{4}$ an inconsistency is found which requires the unphysical result that $\lambda_{1}$ be negative. The only reasonable explanation, or escape, is that $\lambda_{4}$ must be zero, as this allows the $\hat{B}$ term to have significant influence at large negative $\xi$. In fact,

$$
\hat{F} \sim \hat{B}-\frac{2 \hat{B}^{3}}{\lambda_{1}^{2}}|\xi|^{-4 / 3},
$$

from the effective balance between $\hat{B}^{3} \hat{P}^{\prime}$ and higher-order terms in $\hat{F}-\hat{B}$ in $(5.13 c)$. The reasoning above also requires $\alpha c_{1}$ to be positive, corresponding to a rightwardmoving touchdown. The trend of $\hat{F}$ slowly increasing towards $\hat{B}$, in (5.14), is observed in the numerical treatment below.

The required numerical solution of $(5.13 b-e)$ was then obtained and is shown in figure 5 , with $\Gamma, \hat{B}, \alpha c_{1}$ normalized to $1 / 12,4 / 5,1$, respectively. To help determine the solution, given that an arbitrary shift in $\xi$ leaves $(5.13 b-e)$ unchanged, the value of $\hat{F}$ was set at a particular level greater than $\hat{B}$ at one specific $\xi$ station. We note that, even so, a convergent iterative method for the numerical solution proved difficult to find, with most methods tending to approach spurious singular or related solution forms. This difficulty is felt to be connected with seeking the separatrix-like behaviour in $(5.13 d, e)$; a similar response is present in the supersonic counterpart, mentioned earlier in this section, for which the counterpart of $(5.13 b-e)$ allows a phase-plane analysis that indicates the sensitivity of deriving the necessary separatrix solution with many other distinct solutions nearby. The convergent method that was found to work in our case was based on an iterative method in which $(5.13 c)$ 

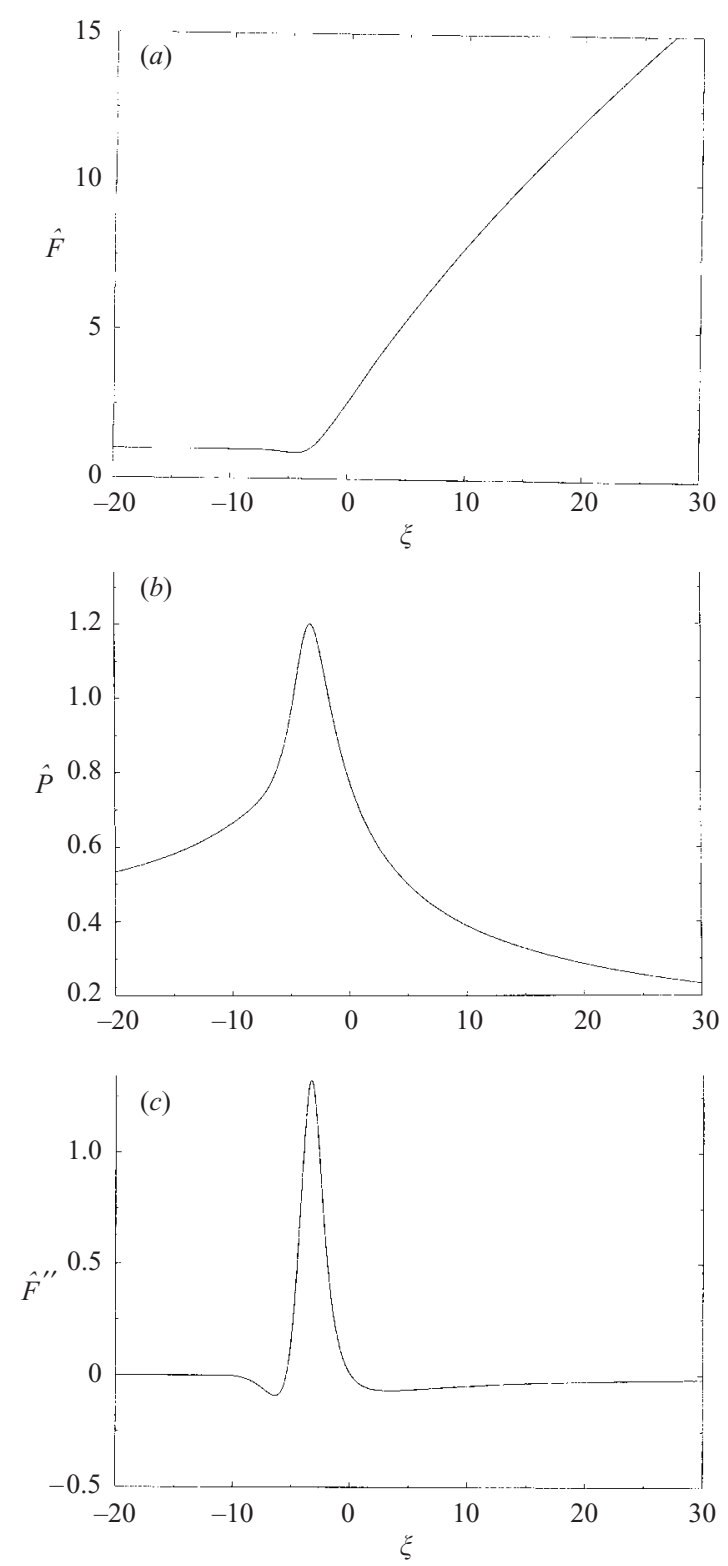

FIGURE 5. Solution of the touchdown problem $(5.13 b-e)$, in normalized form. (a) $\hat{F},(b) \hat{P},(c)$ right-hand side of $(5.13 b)$.

was substituted into (5.13b) to yield a nonlinear equation for $\hat{F}$ only. The principalvalue integral was discretized with a formula developed in $\mathrm{Li}$ (1998) while first and second derivatives were central differenced. The resulting nonlinear discrete system with asymptotes imposed sufficiently far to the left and right was solved by NewtonRaphson iterations, as for a two-point boundary-value problem, giving the result in figure 5 .

The constant $\hat{B}$ in $(5.13 c)$ represents the main distinctive feature in the shorter-scale region compared with the more outer system $(5.10 a, b)$. It is linked with the temporal 
variation of the integral in $X$ of $F$, from (2.5), thus signifying a global influence. In the same vein, the absence of unbounded growth in $\hat{F}$ as $\xi \rightarrow-\infty$ and hence the mildness of $F$ as $\eta$ approaches $-c_{1}$ from the left suggests there might be a solution of $(5.10 a, b)$ in which $\widetilde{F}$ is identically zero for all $\eta$ below $-c_{1}$ and in a sense condition (5.10a) no longer applies for $\eta<-c_{1}$, but instead affects higher-order contributions. The latter is in line with the balancing of higher-order terms as $\hat{F}$ approaches $\hat{B}$ at large negative $\xi$ as described following (5.13e). Such a one-sided outer solution is found to have

$$
\begin{gathered}
\tilde{F}=\lambda_{1}\left(\eta+c_{1}\right)^{2 / 3}, \quad \tilde{P}=\lambda_{2}\left(\eta+c_{1}\right)^{-1 / 3} \text { for } \eta>-c_{1}, \\
\tilde{F}=0, \quad \tilde{P}=\lambda_{3}\left|\eta+c_{1}\right|^{-1 / 3} \text { for } \eta<-c_{1}, \\
\alpha=1 .
\end{gathered}
$$

This analytical form satisfies $(5.10 a, b)$ exactly for all $\eta \neq-c_{1}$, and hence is also an exact solution of the original system (2.3), (2.5), while on a shorter scale the solution of $(5.13 b-e)$ in figure 5 provides the necessary local smoothing.

The account of the touchdown as $T \rightarrow T_{0}-$ in (5.9)-(5.15c) agrees well with the computed behaviour in the time-marching results of figures 3 and 4 in all cases at least in qualitative terms, viewed locally, especially concerning the increasing pressures as the air gap closes. The travelling form $\left[X-X_{0} \sim c_{1}\left(T-T_{0}\right)\right]$ of the touchdown's inner region, giving the major local adjustment, is clearly in keeping with the sideways movement of $F, P$ in the time-marching results just prior to touchdown in figures 3 and 4 . This agreement is reinforced by the zero- $\tilde{F}$ solution in $(5.15 b)$, which is in line with the slow (fast) variation of $F$ to the left (right) of touchdown in all the time-marching results of figures 3 and 4 , in contrast with the fast variation of $P$ throughout. Moreover, since the $\hat{B}$ term generates an $F$ of order $\left(T_{0}-T\right)$ to the power $2 n / 3$ from (5.12), (5.15c), a matching with a regular form of $F$ at 0 (1) values of $X-X_{0}$ would point to the value $n=3 / 2$, which is acceptable for both the local analysis and the time-marching computations. The touchdown described in (5.9)-(5.15c) thus appears to be a universal form, in which the dynamic balance of viscous-lubrication (air)/inviscid (water) pressures is maintained as the interface locally moves even closer to the solid surface, generating a high local pressure peak. Again, the local non-symmetry of each touchdown is responsible for the occurrence of a double peak, either side of the symmetry position, in the symmetric cases.

\section{Further comments and conclusion}

\subsection{Cushioning and touchdown}

The present theoretical study on air-cushioning has mainly considered the balance of viscous lubrication forces in the air against inviscid forces in the water. It should be noted immediately that, under the type of conditions studied so far, this cushioning seems unlikely to significantly delay or avoid impact of the water on the solid wall (say), according to the findings on touchdown in the preceding section. Travellingwave solutions for instance with no touching down cannot occur as linearizations of the fundamental system (2.3), (2.5), or with wall movement, although they can for some simplified models (and also for the different settings in King \& Tuck (1993) and related papers). 


\subsection{Connection with experiments}

The common occurrence of approaching touchdown in all the present spatio-temporal cases and the presumed subsequent formation of successive air pockets may be connected with the experimental observations mentioned in $\S 1$, in particular those in Howison et al., Wilson, Nethercote et al., Driscoll \& Lloyd and Lesser \& Field. Evidence of significant pre-impact adjustment in water-droplet shape can also be discerned in figures $2(a, c, f, h)$ of Liow's (2001) experiments, where, although that paper concentrates more on post-impact properties, typically a water droplet of radius 1-2 $\mathrm{mm}$ has an approach speed of $1-4 \mathrm{~m} \mathrm{~s}^{-1}$ prior to impact, which gives Reynolds numbers within the present subcritical range. In some configurations, compressibility must become important. For an example considered in $\S 3$, of the fraction $\delta=$ $1 / 100$ ( $\delta$ being the relative air-gap width), with a body of water of radius $1 \mathrm{~cm}$ approaching a wall at speed $V=2 \mathrm{~m} \mathrm{~s}^{-1}$, the local Mach number in the air flow is estimated to be 0.3 or so. This simple-minded estimate follows from the ratio of a typical velocity $u_{2}^{*}$ in the air to the typical local sound speed $a_{2}^{*}$; the former is $V / \delta$ from $\S 2$, giving $200 \mathrm{~m} \mathrm{~s}^{-1}$, while $a_{2}^{*}$ is about $700-800 \mathrm{~m} \mathrm{~s}^{-1}$ evaluated from the square root of $\gamma p_{2}^{*} / \rho_{2}$, with $p_{2}^{*} \sim \rho_{1} V^{2} / \delta$ from $\S 2$ additional to the standard atmospheric pressure of approximately $1.01 \times 10^{6}$ c.g.s. units and with $\gamma=1.4$. The other numerical examples with slightly larger values of $\delta$ considered in $\S 3$ yield lower Mach numbers. If compressibility does indeed enter significantly, however, during the lubrication/inviscid stage of the present study it affects the interaction equations if not the whole flow structure; see Korobkin $(1997,1999)$ in the inviscid regime. Alternatively, the compressible effect seems perhaps more likely to become significant when even higher pressures are induced as the approach to touchdown continues (figures 3-5), but as a localized effect at first then. If the latter occurs, then clearly the present stage provides a background flow quite distinct from that usually assumed in the compressible flow studies so far. The presence of under-pressures in a few cases, e.g. figure $4(d)$, raises also the possibility of cavitation. Along with this, however, it is interesting to note finally here the air entrapments and in particular the induced pressure measurements shown in figure 9 of Lesser \& Field's (1983) review of liquid impacts. The experimental pressures are attributed to compressible effects but in fact are qualitatively similar, in their pronounced double-peaked form, to those indicated by the current work (figures 3 and 4) based on viscous-lubrication/inviscid effects.

\subsection{Enhanced interactions}

The assumptions of the inviscid/lubrication theory as investigated in $\S 3$ and in particular the numerical values of the major parameters inherent in the argument indicate a perhaps surprisingly wide range of applicability. Moreover, although the crossover from inviscid/lubrication dynamics to inviscid/inviscid dynamics hinges on a Reynolds number $r e_{1}$ or $R e_{1}$ (see figure 2) which is often large for air and water (a critical value of $R e_{1}$ over $10^{7}$ is recorded in $\S 3$ ), it is interesting that the complete range is covered by having the unsteady boundary-layer equations holding in the air gap. These fuller equations yield $(2.5)$ and $(2.10 a, b)$ as special cases. Similarly, the approaching touchdown phenomenon studied in $\S 5$ and that indicated numerically in Wilson (1991) arise then as special cases, while the finite-time breakup, change of scales and local vortex creation of Smith (1988), Smith, Bowles \& Walker (2000) are interesting possibilities for the fuller system in the air cushion. The time scale involved is always relatively short. The issue of post-impact evolution is also of interest in that broader system. 


\subsection{Extensions}

Several other interactions are indicated by the work. (i) If the density and viscosity ratios are treated as of order unity (e.g. Timoshin 1997) then the thin water and air layers in the specific context of $\$ 4.1$ interact well before impact occurs; again the unsteady boundary-layer equations apply. (ii) With an $O(1)$ ratio of kinematic viscosities but small density ratio, the air flow (see $\S 4.2$ ) in that same context is controlled again by the unsteady boundary-layer equations but with prescribed gap thickness. This again raises the issue of the full finite-time breakup mentioned in the previous paragraph. (iii) If the density ratio in $\S 4$ is specifically of order $\epsilon$, so that the parameter $\Delta$ is of order unity, then another new interaction arises between the air boundary layer and the inviscid bulk of the water, to determine the scaled shape $f_{2}$, prior to impact. The interaction there becomes more subtle still if the density ratio is increased. (iv) The influence of compressibility on the present interactions seems of theoretical and practical relevance. (v) Pronounced gravity effects as indicated within $\S 2.2$ may be worth studying, even though they do correspond to extreme Froude numbers.

\subsection{To conclude}

As far as the work in this paper is concerned, the question raised of when and how air cushioning occurs is answered by the ranges of the scalings in $(2.6),(2.7 a-d)$, together with the local flow structure described in $\S 2$ for a general local setting and in $\S 4$ for a specific global setting. This is for incompressible fluids without surface tension. In particular, air-lubrication cushioning holds for a global Reynolds number in the water below about $10^{7}$, an unsteady boundary-layer cushion for about $10^{7}$, and formally an inviscid cushioning effect above $10^{7}$. Thus the lubrication case has a wide range of practical validity. This case yields the problem solved in $\S 5$. The results show that the air-pressure feedback tends to be at its strongest initially near the head (the minimum gap) of the parabolic shape of the air-water interface approaching a wall, as might be expected physically, and so decelerates or even reverses the motion of the interface there. Similar deceleration occurs for other incident shapes or configurations. The raised pressure in the middle then tends to drive fluid to the sides. Indeed, there is a tendency towards touchdown of the water onto the wall on either side of the original head, a phenomenon which suggests subsequent trapping of air pockets (although no doubt some incident configurations might avoid the occurrence of the touchdown altogether); the touchdown is accompanied by enhanced local pressures, yielding a double peak typically as in figures 3 and 4 . The touchdown phenomenon, the very high induced pressure peaks, their connection with experiments, and the $O\left(10^{7}\right)$ crossover Reynolds number are perhaps the prime results of this work.

Thanks are due to Drs M. J. Cooker, S. D. Howison, J. R. Ockendon, S. N. Timoshin, S. K. Wilson and the referees for their interest and comments and to the Leverhulme Trust for support for L. L.

\section{Appendix. Asymptotic expansions for the interaction of $\S 2$}

The Navier-Stokes equations, (2.1), written in non-dimensional form are

$$
\left[\frac{\partial}{\partial \tilde{\tilde{t}}}+\tilde{\tilde{\boldsymbol{u}}}_{\boldsymbol{n}} \cdot \tilde{\tilde{\nabla}}\right] \tilde{\tilde{\boldsymbol{u}}}_{\boldsymbol{n}}=-H_{n} \tilde{\tilde{\nabla}} \tilde{\tilde{p}}_{n}-\left(0, \frac{1}{\mathscr{F}}\right)+\frac{h_{n}}{r e_{1}} \tilde{\nabla}^{2} \tilde{\tilde{\boldsymbol{u}}}_{\boldsymbol{n}},
$$


in the water $(n=1)$ and air $(n=2)$, with continuity requiring $\underset{\tilde{\tilde{\nabla}}}{\tilde{\nabla}} \cdot \tilde{\tilde{\boldsymbol{u}}}_{\boldsymbol{n}}=0$. Here $H_{1}=$ $h_{1}=1, H_{2}=\rho_{1} / \rho_{2}, h_{2}=v_{2} / v_{1}, \tilde{\tilde{\boldsymbol{u}}}_{n}=\left(u_{n}^{*}, v_{n}^{*}\right) / V, \tilde{\tilde{t}}=V t^{*} / \ell, \tilde{\nabla}$ denotes the operator $(\partial / \partial X, \partial / \partial Y)$ with $Y=y^{*} / \ell$, and $\tilde{\tilde{p}}_{n}=p_{n}^{*} /\left(\rho_{1} V^{2}\right)$. We observe that unlike (4.1) the local Reynolds number $r e_{1}$ in the water appears here.

The expansion of the solution in the majority of the water motion is

$$
\tilde{\tilde{u}}_{1}=\left(u_{1}, v_{1}\right)+\cdots, \quad \tilde{\tilde{p}}_{1}=\delta^{-1} p_{1}+\cdots
$$

with $u_{1}, v_{1}, p_{1}$ and the coordinates $X, Y$ of $O(1)$, while $\tilde{\tilde{t}}=\delta T$ with $T$ of $O(1)$. The expansion (A 2), coupled with the assumptions $(2.7 a-d)$ and substituted into (A 1) with $n=1$, yields the governing equations $(2.2 a-c)$ at leading order provided that $u_{1}^{*}, v_{1}^{*}, p_{1}^{*}, x^{*}, y^{*}, t^{*}, \rho_{1}$ are replaced there by $u_{1}, v_{1}, p_{1}, X, Y, T, 1$, respectively. Similarly, the interfacial boundary conditions $\operatorname{read} v_{1} \rightarrow \partial F / \partial T, p_{1} \rightarrow P(X, T)$ as $Y \rightarrow 0+$, analogous to $(2.2 d)$, with the interface being at $Y=\delta F(X, T)+\cdots$. Hence, the first relation (2.3) between $F$ and $P$ is obtained.

The expansion of the flow solution in the air gap has the form

$$
\tilde{\tilde{\boldsymbol{u}}}_{2}=\left(\delta^{-1} \hat{u}, \hat{v}\right)+\cdots, \quad \tilde{\tilde{p}}_{2}=\delta^{-1} p_{2}+\cdots
$$

where $\hat{u}, \hat{v}, p_{2}, X$ are now $O(1)$, but $Y=\delta \hat{y}$ with $\hat{y}$ of $O(1)$. This holds together with $(2.7 a-d)$ again. Upon substitution into (A 1) with $n=2$, (A 3) implies that the $Y$-momentum component reduces to $\partial p_{2} / \partial \hat{y}$ being zero, so that $p_{2}=P(X, T)$, while the $X$-momentum component gives $(2.4 a, b)$ with $u_{2}^{*}, v_{2}^{*}, p_{2}^{*}, x^{*}, y^{*}, \mu_{2}$ replaced there by $\hat{u}, \hat{v}, P, X, \hat{y}, 1$ in turn, to leading order. The boundary conditions here are as given in the equations immediately before (2.5). It follows that the second relation, (2.5), between $F$ and $P$ holds.

\section{REFERENCES}

BarannyK, L. L. \& Papageorgiou, D. T. 2002 Fully nonlinear gravity-capillary solitary waves in a two-fluid system of finite depth. J. Engng Maths 42, 321-339.

CoOKer, M. J. 1997 Recent numerical calculations of two-dimensional jets induced by breaking-wave impact; a comment. Phil. Trans. R. Soc. A 355, 687-689.

Cooker, M. J., Weidman, P. D. \& Bale, D. S. 1997 Reflection of a high-amplitude solitary wave at a vertical wall. $J$. Fluid Mech. 342, 141-158.

Davis, S. H. 2000 Interfacial fluid dynamics. In Perspectives in Fluid Dynamics, chap. 1 (ed. G. K. Batchelor, H. K. Moffatt \& M. G. Worster). Cambridge University Press.

Driscoll, A. \& Lloyd, A. 1982 Slamming experiments - description of facilities and details of impact pressure results. Rep. R82002, AMTE (Haslar).

Duffy, B. R. \& WiLson, S. K. 1999 Thin film and curtain flows on the outside of a rotating cylinder. J. Fluid Mech. 394, 29-49.

FitT, A. D. \& Pope, M. P. 2001 The unsteady motion of two-dimensional flags with bending stiffness. J. Engng Maths 40, 227-248.

Howison, S. D., Ockendon, J. R. \& Oliver, J. M. 2002 Deep- and shallow-water slamming at small and zero deadrise angles. J. Engng Maths 42, 373-388.

Howison, S. D., Ockendon, J. R. \& Wilson, S. K. 1991 Incompressible water-entry problems at small deadrise angles. J. Fluid Mech. 222, 215-230.

JONES, M. A. 2000 Mechanisms in wing-in-ground-effect dynamics. PhD thesis, University of London.

Jones, M. A. \& SMith, F. T. 2003 Fluid motion for car undertrays in ground effect. J. Engng Maths (in press).

Joseph, D. D. \& Preziosi, L. 1987 Stability of rigid motions and coating films in bicomponent flows of immiscible liquids. J. Fluid Mech. 185, 323-351.

KANG, Y. \& VANDEN-BroeCK, J.-M. 2000 Gravity-capillary waves in the presence of constant vorticity. Eur. J. Mech. B 19, 253-268. 
Kelmanson, M. A. 1995 Theoretical and experimental analyses of the maximum-supportable fluid load on a rotating cylinder. J. Engng Maths 29, 271-285.

KING, A. C. \& Tuck, E. O. 1993 Thin fluid layers supported by surface traction. J. Fluid Mech. 251, 709-718.

King, A. C., Tuck, E. O. \& Vanden-Broeck, J.-M. 1993 Air-blown waves on thin viscous sheets. Phys. Fluids A 5, 973-978.

Korobkin, A. A. 1997 Asymptotic theory of liquid-solid impact. Phil. Trans. R. Soc. Lond. A 355, 507-522.

Korobkin, A. A. 1999 Shallow water impact problems. J. Engng Maths 35, 233-250.

Korobkin, A. A. \& Peregrine, D. H. 2000 The energy distribution resulting from an impact on a floating body. J. Fluid Mech. 417, 157-181.

Kriegsmann, J. J., Miksis, M. J. \& Vanden-Broeck, J.-M. 1998 Pressure driven disturbances on a thin viscous film. Phys. Fluids 10, 1249-1255.

Lesser, M. B. \& Field, J. E. 1983 The impact of compressible liquids. Annu. Rev. Fluid Mech. 15, 97-122.

LI, L. 1998 Numerical study of nonlinear evolution equations, using compact differencing. PhD thesis, University of London.

Li, L., Papadopoulos, D. P., Smith, F. T. \& Wu, G.-X. 2001 Water flow due to rapid part-submerged body movement. Proc. IUTAM Symp. on free-surface flows, Birmingham, July 2000 (ed. A. C. King, Y. D. Shikhmurzaev), pp. 177-185. Kluwer.

Li, L., Papadopolous, D. P., Smith, F. T. \& Wu, G.-X. 2002 Rapid plunging of a body partly submerged in water. J. Engng Maths 42, 303-319.

Liow, J. L. 2001 Splash formation by spherical drops. J. Fluid Mech. 427, 73-105.

MCKinley, I. S. \& WiLSON, S. K. 2001 The linear stability of a ridge of fluid subject to a jet of air. Phys. Fluids 13, 872-883.

Miksis, M. J. \& VAnden-Broeck, J.-M. 1999 Self-similar dynamics of a viscous wedge of fluid. Phys. Fluids 11, 3227-3231.

Nethercote, W. C. E., Mackay, M. \& Menon, B. 1986 Some warship slamming investigations. DREA Tech. Memo. 86/206.

Oron, A., Davis, S. H. \& Bankoff, S. G. 1997 Long-scale evolution of thin films. Rev. Mod. Phys. 69, 931-980.

Papadopoulos, D. P. 2000 On the unsteady boundary layer and wake of a flat plate, and modelling water flow near a ship-side. PhD thesis, University of London.

Purvis, R. 2002 Rotor blade flows and ground effect. PhD thesis, University of London.

Qú́RÉ, D. 1999 Fluid coating on a fibre. Annu. Rev. Fluid Mech. 31, 347-384.

ReIn, M. 1993 Phenomena of liquid drop impact on solid and liquid surfaces. Fluid Dyn. Res. 12, 61-93.

SмITH, F. T. 1988 Finite-time breakup can occur in any unsteady interacting boundary layer. Mathematika 35, 256-273.

Smith, F. T., Bowles, R. I. \& Walker, J. D. A. 2000 Wind-up of a spanwise vortex in deepening transition and stall. Theor. Comput. Fluid Dyn. 14, 135-165.

Timoshin, S. N. 1997 Instabilities in a high-Reynolds-number boundary layer on a film-coated surface. J. Fluid Mech. 353, 163-195.

TucK, E. O. \& BENTwich, M. 1983 Sliding sheets: lubrication with comparable viscous and inertia forces. J. Fluid Mech. 135, 51-69.

VANDEN-BroecK, J.-M. 2001 Damped waves generated by a moving pressure distribution. Eur. $J$. Appl. Maths 12, 357-366.

Vanden-Broeck, J.-M. \& Miloh, T. 1996 The influence of a layer of mud on the train of waves generated by a moving pressure distribution. J. Engng Maths 30, 387-400.

Walkden, M. J., Wood, D. J., Bruce, T. \& Peregrine, D. H. 2001 Impulsive seaward loads induced by wave overtopping on caisson breakwaters. Coastal Engng 42, 257-276.

WARD, S. N. 2001 Landslide tsunami, J. Geophys. Res. 100, 24 487-24 498.

WARD, S. N. \& DAY, S. 2001 Cumbre Vieja volcano - potential collapse and tsunami at La Palma, Canary Islands. Geophys. Res. Lett. 28, 3397-3400.

Weidner, D. E., Schwartz, L. W. \& Eres, M. H. 1997 Simulation of coating layer evolution and drop formation on horizontal cylinders. J. Colloid Interface Sci. 187, 243-258. 
Weiss, D. A. \& Yarin, A. L. 1999 Single droplet impact onto liquid films: neck distortion, jetting, tiny bubble entrainment, and crown formation. J. Fluid Mech. 385, 229-254.

WiLson, S. K. 1991 A mathematical model for the initial stages of fluid impact in the presence of a cushioning fluid layer. J. Engng Maths 25, 265-285.

Wilson, S. K. \& Duffy, B. R. 1998 On lubrication with comparable viscous and inertia forces. Q. J. Mech. Appl. Maths 51, 105-124.

Wood, D. J. \& Peregrine, D. H. 2000 Wave impact on a wall using pressure-impulse theory. II: porous berm. J. Waterway, Port, Coastal, Ocean Engng ASCE 126, 191-195.

Wood, D. J., Peregrine, D. H. \& Bruce, T. 2000 Wave impact on a wall using pressure-impulse theory. I: trapped air. J. Waterway, Port, Coastal, Ocean Engng ASCE 126, 182-190.

YARIN, A. L. \& WeISS, D. A. 1995 Impact of drops on solid surfaces: self-similar capillary waves and splashing as a new type of kinematic discontinuity. J. Fluid Mech. 238, 141-173.

Zhao, R. \& Faltinsen, O. 1993 Water entry of two-dimensional bodies. J. Fluid Mech. 246, 593-612.

Zhu, Y., Oguz, H. N. \& Prosperetti, A. 2000 On the mechanism of air entrainment by liquid jets at a free surface. J. Fluid Mech. 404, 151-177. 